\title{
Building up an Inertial Navigation System Using Standard Mobile Devices
}

\author{
Marco Gunia, Yan Wu, Niko Joram and Frank Ellinger \\ Chair of Circuit Design and Network Theory (CCN), Technische Universität Dresden, Dresden 01062, Germany
}

\begin{abstract}
This paper presents the development of a PNS (Pedestrian Navigation System), which utilizes accelerometer, gyroscope and magnetometer data to enable accurate positioning. Therefore, the sensor basics as well as the mathematics regarding reference frames and coordinate transformations are introduced initially. Particular focus is given to quaternions, since they provide a performance-effective means to execute rotations. In great detail the two distinct approaches for a PNS are introduced, i.e. INS (inertial navigation systems) and PDR (pedestrian dead reckoning). For each, a comparison of state-of-the-art techniques is presented. Special attention is paid to orientation estimation and stance phase detection. Our system combines the most promising techniques and describes improvements, whose usefulness becomes obvious in our experiments. We have applied our PNS in five different test scenarios. For the most complex rectangular-shaped use case, we achieve on average error of $1.66 \%$ with regard to the total travelled distance, which is superior to other recent PNS utilizing comparable sensors.
\end{abstract}

Key words: Positioning, accelerometer, gyroscope, magnetometer, quaternion, INS, PDR, complementary filter.

\section{Introduction}

Nowadays much research activities are devoted to provide hybrid localization. A related topic, which is a first step towards this goal, is seamless positioning. Here, multiple individual localization systems are distributed spatially, for instance in different rooms for indoor applications. The objective is to enable localization by selecting the positioning system available on the very spot without informing the user that a transition to another system might have taken place. However, these areas could be non-overlapping, thus generating black sectors, where positioning is impossible. One alternative to bridge these spots is by using dead reckoning systems, as explained below.

Loosely speaking, navigation systems can be classified into position fixing and dead reckoning techniques. For the former, the object to be localized is evaluated with regard to known features in the environment. As an example, the position of a GPS

Corresponding author: Marco Gunia, Dipl.-Inf. Dipl.-Ing., research associate at chair of Circuit Design and Network Theory, research fields: seamless indoor and outdoor positioning. receiver can be determined with the help of satellites, whose positions are known. However, this category also includes most indoor positioning systems. For instance, a Wireless Local Area Network receiver in a smartphone could be localized by measuring the signal strength regarding multiple access points. Another example is an ultra-wideband radar system consisting of multiple base stations, which determine the distance to a mobile entity, respectively, by measuring the time of flight [1]. Another famous approach is FMCW (frequency modulated continuous wave) radar, where the distance is computed by applying frequency chirps and utilizing time difference of arrival [2]. In contrast, dead reckoning systems do not require any knowledge about the environment. Moreover, they do not depend on any remote hardware. Instead, they employ user attached sensors, to determine the position in respect to a starting point. Usually, accelerometers, gyroscopes and magnetometers are utilized.

Within this paper, the steps necessary to build up a PNS are presented, where only the aforementioned sensors are used, which are all available in modern hand-held devices. A particular focus is on the basics 
and on related work. State-of-the art approaches for INS and PDR are analysed and auspicious candidates are selected. It turns out that orientation estimation and stance phase detection significantly influence the results; thus strong techniques for both are elaborated.

The rest of this paper is organized as follows. Section 2 introduces the theoretical foundations, e.g. sensor basics, reference frames, and coordinate transformations. Especially, quaternions are introduced, which are a performance effective means for executing rotations. Related work is presented in Section 3. In particular, the two main techniques are described and comparisons of approaches from literature are given. As already stated, accurate orientation is a key factor. Multiple variants are presented in Section 4. On this basis, our PNS is introduced in Section 5. Manifold experiments are listed in Section 6. Here, our system is evaluated in regard to other state-of-the art approaches. Moreover, unresolved issues are identified. The last Section 7 concludes the paper.

\section{Basics}

This section presents terminology and essential basics. After presenting the sensors used, reference frames and some mathematical principles regarding coordinate system transformations are introduced. Finally, the human gait cycle is illustrated.

\subsection{Sensors}

Although today's mobile end devices cover manifold sensors, only accelerometers, gyroscopes and magnetometers are employed in this paper and are introduced below. Alternative sensors are not considered. The reasons for this are twofold. On the one hand, these sensors provide raw data, whereas other sensors being software entities, which utilize these data to perform some form of preprocessing. On the other hand, sensors like barometers do only provide very coarse information for positioning [3].

Accelerometers measure the acceleration of a rigid body within an inertial navigation system, based on Newton's second law of motion, i.e.

$$
\boldsymbol{F}=m \cdot \boldsymbol{a}
$$

Fig. 1a shows the principle. The mass is kept in balance in such a way that an equilibrium of forces arises. Due to any additional external acceleration, the mass is relocated, to finally create another equilibrium. With the help of the measurement scale, the value of the external acceleration can be determined. A realisation of this principle as MEMS (micro electro mechanical system) is shown in Fig. 1b. Here, the values of the capacitances $C_{1}$ and $C_{2}$ are changed due to the relocation of the mass as a result of external acceleration. It is worth mentioning again, that the values of accelerometers are referred to an inertial navigation system. Hence, gravity is considered as acceleration by the sensors. To take this into account, the term linear acceleration denotes acceleration where the gravity component is already removed.

Gyroscopes determine the angular velocity. Therefore, the Coriolis effect is applied, which states that a mass $m$ with velocity $\boldsymbol{v}$ experiences a force $\boldsymbol{F}_{C}$ in a reference frame rotating at $\boldsymbol{\omega}$ :

$$
\boldsymbol{F}_{C}=-2 \cdot m(\boldsymbol{\omega} \times \boldsymbol{v})
$$

The principle is shown in Fig. 2a, where a single mass $m$ is stimulated to vibrate along a drive axis. If in this context the gyroscope is rotated, then a secondary vibration is generated along the perpendicular sense axis due to the Coriolis force. The angular velocity can then be derived by measuring this secondary rotation [6]. One realisation for MEMS is based on a comb drive which is set into vibration (see

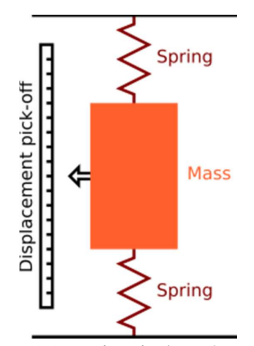

(a) Principle [4]

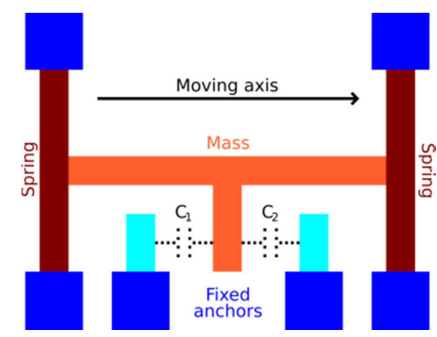

(b) MEMS realization [5]
Fig. 1 Accelerometer. 


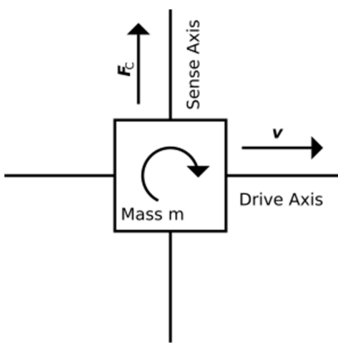

(a) Principle [6]

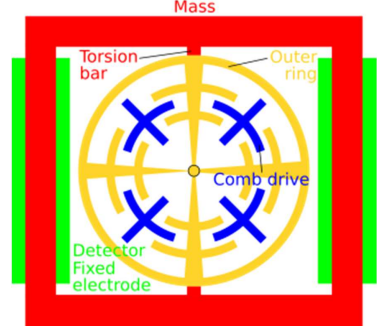

(b) MEMS realization [5]
Fig. 2 Gyroscope.

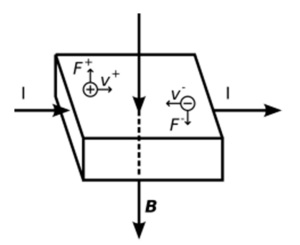

(a) Principle

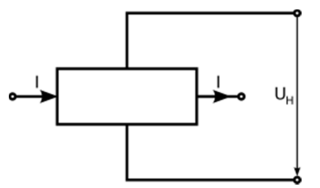

(b) Hall voltage
Fig. 3 Magnetometer [8].

Fig. 2b). The rotating axis points into the paper. Due to motion, the distance between the plates of the capacitor, formed between the green detector and the red mass, is changed. This enables to determine the angular velocity.

Magnetometers measure the local magnetic field. Therefore, the Hall effect is utilized, as shown in Fig. 3. Due to the magnetic flux density $B$, moving electrons, which constitute the current $I$ within the conductor, are shifted towards the upper and lower plate, thus generating a Hall voltage $U_{H}$.

For positioning three mutually perpendicular sensors are necessary, respectively. In the following, the term accelerometer, gyroscope or magnetometer will always denote such an entity, respectively. Moreover, as an IMU (inertial measurement unit) we specify an entity containing accelerometer and gyroscope.

In Ref. [6] a thorough analysis of different errors sources, which affect the performance of MEMS sensors is given. The main error sources are:

- Constant bias;

- Thermo-mechanical white noise;

- Flicker noise;

- Calibration errors.

The constant bias is the average output value, when no input quantity is present. It is the offset part from the true value. The sensor output is perturbed by white noise, which fluctuates at a much higher frequency than the sampling rate of the sensors. Within the electronic components of the sensors, Flicker noise arises, which influences the bias at low frequencies. Moreover, calibration errors are caused by errors in the scale factors. Their influences are only visible if an input is present. According to Ref. [6], constant bias and white noise are the most important sources for accelerometer and gyroscope MEMS.

\subsection{Reference Frames}

Within this paper all reference frames are orthogonal, right-handed coordinate frames. The local navigation frame and the body frame are the two main frames being applied. They are introduced below. For the local navigation frame $\mathrm{N}$, the origin is the stand-point of the user. The three orthogonal axes are aligned in the directions north, east and down as shown in Fig. 4a. In contrast, the body frame B is aligned with the body in consideration. The origin is one point of the body, e.g. the centre of mass. The x-axis (also known as roll-axis) points forward, the $y$-axis (also known as pitch-axis) points to the right, and the $\mathrm{z}$-axis (also known as yaw-axis) points downward (see Fig. 4b). For our considerations, the origins between both frames are always the same.

\subsection{Coordinate Transformation}

In this subsection the relationships for coordinate transformations are summarized. Proves can be found in Refs. [4, 9]. By means of ${ }^{A} \boldsymbol{v}$ a vector $\boldsymbol{v}$ is denoted within the coordinate frame $A$. The representation of this vector in another coordinate frame $B$, i.e. ${ }^{B} \boldsymbol{v}$, can be accomplished with the help of a coordinate transformation.

$$
{ }^{B} \boldsymbol{v}=\mathrm{C}_{A}^{B} \cdot{ }^{A} \boldsymbol{v}
$$

The transformation matrix $\mathrm{C}_{A}^{B}$ is the DCM (direction cosine matrix), where the element in the $i$-th row ( $i=1,2,3)$ and $j$-th column $(j=1,2,3)$ is the cosine of the angle between the $\mathrm{i}$-axis of the $A$ frame 


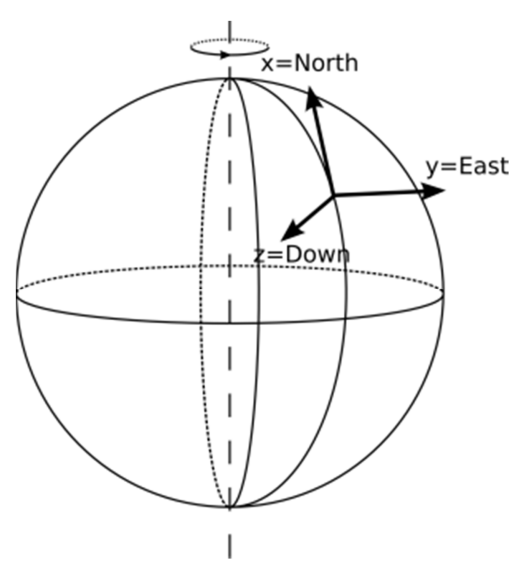

(a) Local navigation frame

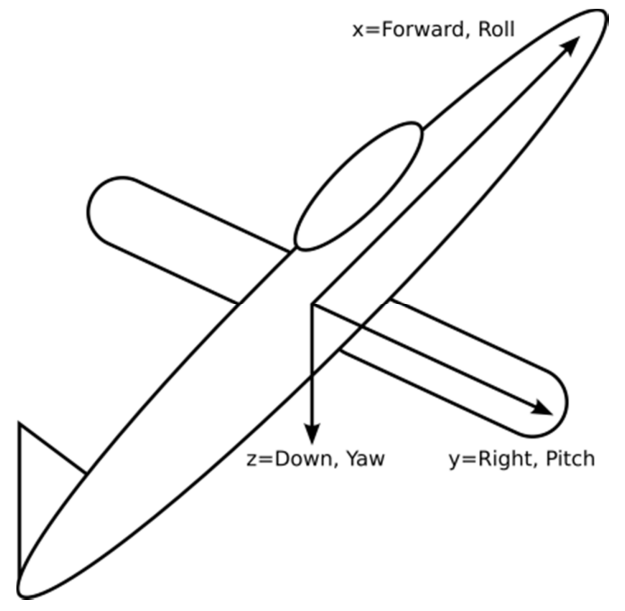

(b) Body frame

Fig. 4 Reference frames [9].

and the $\mathrm{j}$-axis of the $B$ frame [9]:

$$
\mathrm{C}_{A}^{B}=\left(\begin{array}{lll}
c_{11} & c_{12} & c_{13} \\
c_{21} & c_{22} & c_{23} \\
c_{31} & c_{32} & c_{33}
\end{array}\right)
$$

The inverse transformation can be realized by

$$
{ }^{A} \boldsymbol{v}=\mathrm{C}_{B}^{A} \cdot{ }^{B} \boldsymbol{v}
$$

where [9]

$$
\mathrm{C}_{B}^{A}=\left(\mathrm{C}_{A}^{B}\right)^{T}
$$

Hence, for the transformation between body frame $B$ and local navigation frame $N$ the following equation applies

$$
{ }^{N} \boldsymbol{v}=\mathrm{C}_{B}^{N} \cdot{ }^{B} \boldsymbol{v}
$$

In addition to the DCM, there are other methods for coordinate transformation, above all Euler angles, the rotation vector and quaternions. Since the first two suffer from ambiguities [9], i.e. the gimbal locks, quaternions are frequently applied for dead reckoning. Compared to DCM, quaternions consist of only 4 elements instead of 9, reducing computational complexity. However, the interpretation of quaternions is more difficult.

Quaternions are hyper complex numbers with four components [9]

$$
\mathfrak{q}=(w, x, y, z)^{T}
$$

where $w, x, y$ and $z$ are all real numbers. It can also be written with the primitive elements $\mathfrak{i}, \mathfrak{j}, \mathfrak{k}$

$$
\mathfrak{q}=w+x \mathfrak{i}+y \mathfrak{j}+z \mathfrak{k}
$$

or as a sum of the scalar $w$ and a vector $(x, y, z)^{T}$ :

$$
\mathfrak{q}=w+(x, y, z)^{T}
$$

To distinguish quaternions from vectors, we employ Gothic characters. Addition of quaternions is performed for each element. Multiplication $\otimes$ of two quaternions is based on the definition of the multiplication for the primitive elements, i.e.

$$
\begin{aligned}
& \mathfrak{i} \otimes \mathfrak{i}=\mathfrak{j} \otimes \mathfrak{j}=\mathfrak{k} \otimes \mathfrak{k}=-1 \\
& \mathfrak{i} \otimes \mathfrak{j}=-\mathfrak{j} \otimes \mathfrak{i}=\mathfrak{k} \\
& \mathfrak{j} \otimes \mathfrak{k}=-\mathfrak{k} \otimes \mathfrak{j}=\mathfrak{i} \\
& \mathfrak{k} \otimes \mathfrak{i}=-\mathfrak{i} \otimes \mathfrak{k}=\mathfrak{j}
\end{aligned}
$$

For arbitrary quaternions $\mathfrak{q}_{\mathfrak{o}}=w_{0}+x_{0} \mathfrak{i}+y_{0} \mathfrak{j}+z_{0} \mathfrak{k}$ and $\mathfrak{q}_{1}=w_{1}+x_{1} \mathfrak{i}+y_{1} \mathfrak{j}+z_{1} \mathfrak{k}$, the multiplication is defined by applying usual distributive laws and considering Eq. (11), i.e. [12].

$$
\begin{aligned}
\mathfrak{q}_{\circ} \otimes \mathfrak{q}_{1}= & \left(w_{0}+x_{0} \mathfrak{i}+y_{0} \mathfrak{j}+z_{0} \mathfrak{k}\right) \\
\otimes & \left(w_{1}+x_{1} \mathfrak{i}+y_{1} \mathfrak{j}+z_{1} \mathfrak{k}\right) \\
= & \left(w_{0} w_{1}-x_{0} x_{1}-y_{0} y_{1}-z_{0} z_{1}\right) \\
& +\left(w_{0} x_{1}+x_{0} w_{1}+y_{0} z_{1}-z_{0} y_{1}\right) \mathfrak{i} \\
& +\left(w_{0} y_{1}-x_{0} z_{1}+y_{0} w_{1}+z_{0} x_{1}\right) \mathfrak{j} \\
& +\left(w_{0} z_{1}+x_{0} y_{1}-y_{0} x_{1}+z_{0} w_{1}\right) \mathfrak{k}
\end{aligned}
$$

Some further definitions follow Ref. [12]. Let 
$\mathfrak{q}=w+x \mathfrak{i}+y \mathfrak{j}+z \mathfrak{k}$ be an arbitrary quaternion. Its conjugate .* is defined as

$$
\mathfrak{q}^{*}=w-x \mathfrak{i}-y \mathfrak{j}-z \mathfrak{k}
$$

and the norm $\|\cdot\|$ as

$$
\|\mathfrak{q}\|=\sqrt{w^{2}+x^{2}+y^{2}+z^{2}}
$$

A quaternion $\mathfrak{q}$ with $\|\mathfrak{q}\|=1$ is called a unit quaternion, denoted by $\hat{\mathfrak{q}}$. The product of two unit quaternions is again a unit quaternion [11]. Quaternions with first component zero are characterized as pure quaternions. As an example, a vector $\boldsymbol{v} \in \mathbb{R}^{3}$ can be represented as a pure quaternion $\mathfrak{v}_{\mathfrak{p}}$ by

$$
\mathfrak{v}_{\mathfrak{p}}=(0, \boldsymbol{v})^{T}=\left(0, v_{1}, v_{2}, v_{3}\right)^{T} ; \boldsymbol{v}=\left(v_{1}, v_{2}, v_{3}\right)^{T}
$$

Unit quaternions can be used to perform coordinate transformations. Let the operator $L_{\hat{\mathfrak{q}}}(\cdot)$ be defined as

$$
L_{\hat{\mathfrak{q}}}\left(\mathfrak{v}_{\mathfrak{p}}\right)=\hat{\mathfrak{q}} \otimes \mathfrak{v}_{\mathfrak{p}} \otimes \hat{\mathfrak{q}}^{*}
$$

where $\hat{\mathfrak{q}}$ is a unit quaternion, then Eq. (16) characterizes a rotation of the vector $\boldsymbol{v}$, represented as pure quaternion $\mathfrak{v}_{\mathfrak{p}}$ [11]. Specifically, let $\hat{\mathfrak{q}}_{N \leftarrow B}$ denote the coordinate transformation from the body frame to the local navigation frame. Then a vector ${ }^{B} \boldsymbol{v}$ in the body frame, represented as pure quaternion ${ }^{B} \mathfrak{v}_{\mathfrak{p}}$, is transformed into the local navigation frame by [12]

$$
{ }^{N} \mathfrak{v}_{\mathfrak{p}}=\hat{\mathfrak{q}}_{N \leftarrow B} \otimes{ }^{B} \mathfrak{v}_{\mathfrak{p}} \otimes \hat{\mathfrak{q}}_{N \leftarrow B}^{*}
$$

The inverse transformation is accomplished by [11]

$$
\begin{aligned}
{ }^{B} \mathfrak{v} & =\hat{\mathfrak{q}}_{B \leftarrow N} \otimes{ }^{N} \mathfrak{v}_{\mathfrak{p}} \otimes \hat{\mathfrak{q}}_{B \leftarrow N}^{*} \\
& =\hat{\mathfrak{q}}_{N \leftarrow B}^{*} \otimes{ }^{N} \mathfrak{v}_{\mathfrak{p}} \otimes \hat{\mathfrak{q}}_{N \leftarrow B}
\end{aligned}
$$

Conversions between DCM, Euler angels, rotation matrix and quaternions are listed in Ref. [9].

\subsection{Gait-Cycle}

The human gait cycle is shown in Fig. 5. It consists of two alternating phases. In contrast to the swing phase, the stance phase specifies the interval in which the foot in consideration is touching the ground. The stance phase lasts about $60 \%$ of a full gait cycle [10]. According to Fig. 6 this full cycle is also denoted as a stride. In turn, a stride consists of two single steps.

\section{Approaches for PNS}

The generic term Dead Reckoning denotes techniques that calculate position updates from measuring the change in position. Within this section two fundamental approaches are introduced, i.e. the INS (inertial navigation system) and the PDR (pedestrian dead reckoning).

\subsection{INS}

INS can be classified into stable platform systems and strapdown systems. For stable platform systems all the sensors are mounted on a platform which is always aligned to the local navigation frame by using gimbals and motors. Due to their mechanical complexity, they are not utilized in any mobile device. Therefore in the following, only strapdown INS are considered. For these systems, all sensors are directly mounted on a rigid body as shown in Fig. 7. In contrast to stable platform INS, the sensor data are provided within the body frame and need to be converted to the local navigation frame. A flowchart for determining the position with the help of a strapdown system is shown in Fig. 8 and explained below.

Let $\left({ }^{B} w_{x},{ }^{B} w_{y},{ }^{B} w_{z}\right)^{T}$ represent the angular velocity measured by the gyroscope. According to Eq. (15), the associated pure quaternion ${ }^{B} \mathfrak{w}_{\mathfrak{p}}$ is

$$
{ }^{B} \mathfrak{w}_{\mathfrak{p}}=\left(0,{ }^{B} w_{x},{ }^{B} w_{y},{ }^{B} w_{z}\right)^{T}
$$

Since quaternions provide a linear means for manipulating rotations, the updated quaternion representing the coordinate transformation between body frame and local navigation frame $\hat{\boldsymbol{q}}_{N \leftarrow B}$ can approximately be written as Ref. [13]

$$
\mathfrak{q}_{N \leftarrow B} \approx \hat{\mathfrak{q}}_{N \leftarrow B_{0}}+\dot{\mathfrak{q}}_{N \leftarrow B} \cdot \Delta t
$$

Where [13, 14]

$$
\dot{\mathfrak{q}}_{N \leftarrow B}=\frac{1}{2} \cdot \hat{\mathfrak{q}}_{N \leftarrow B_{0}} \otimes^{B} \mathfrak{w}_{\mathfrak{p}}
$$




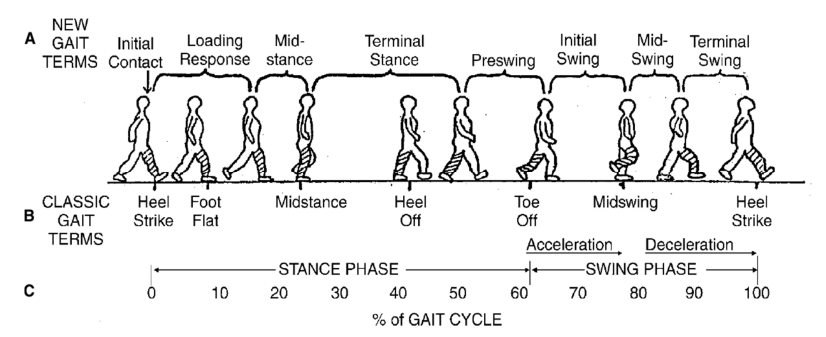

Fig. 5 Human gait cycle [10].

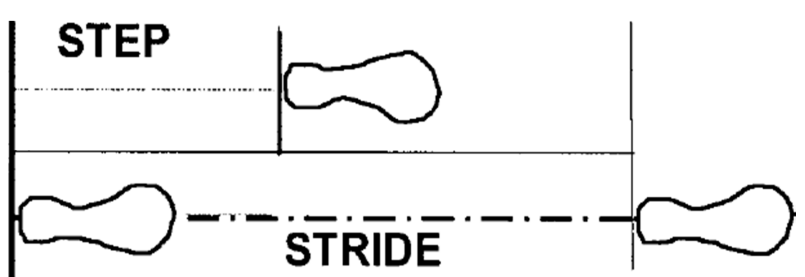

Fig. 6 Step and stride [10].

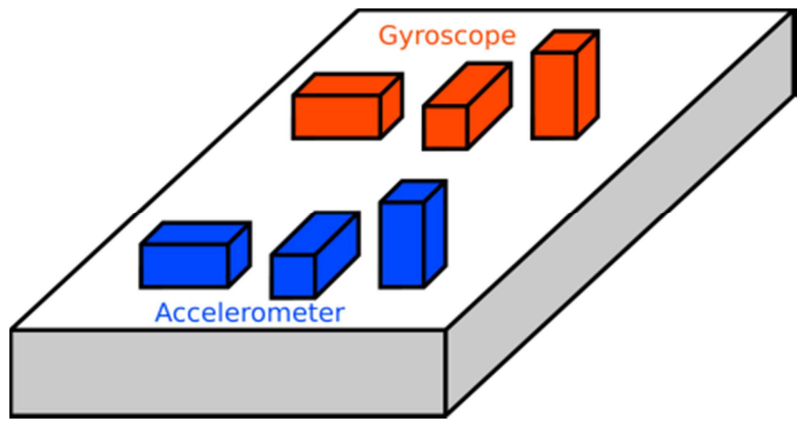

Fig. 7 Strapdown INS.

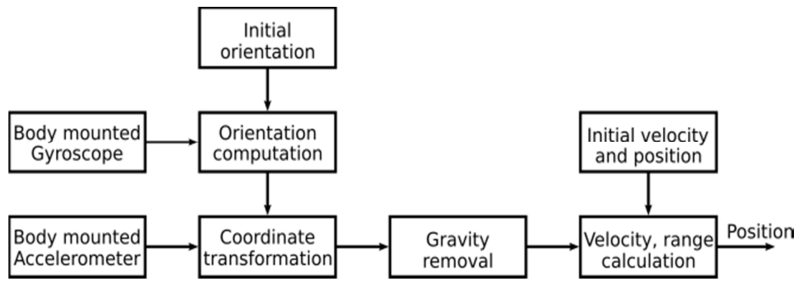

Fig. 8 Flowchart of the strapdown INS.

After this incremental update, the length will not be unity any more. Therefore, normalization is performed

$$
\hat{\mathfrak{q}}_{N \leftarrow B}=\frac{\mathfrak{q}_{N \leftarrow B}}{\left\|\mathfrak{q}_{N \leftarrow B}\right\|}
$$

After accomplishing the orientation computation with Eqs. (20)-(22), the accelerometer measurements ${ }^{B} \boldsymbol{a}$ are transformed in the local navigation frame according to Eq. (17)

$$
{ }^{N} \mathfrak{a}_{\mathfrak{p}}=\hat{\mathfrak{q}}_{N \leftarrow B} \otimes{ }^{B} \mathfrak{a}_{\mathfrak{p}} \otimes \hat{\mathfrak{q}}_{N \leftarrow B}^{*}
$$

where ${ }^{B} \mathfrak{a}_{\mathfrak{p}}$ is the pure quaternion form of ${ }^{B} \boldsymbol{a}$. Afterwards, gravity is removed. This linear acceleration ${ }^{N} \boldsymbol{a}_{\text {lin }}$ is then double integrated to determine the new position ${ }^{N} \boldsymbol{r}$, where the former position ${ }^{N} \boldsymbol{r}_{\mathbf{0}}$ and velocity ${ }^{N} \boldsymbol{v}_{\mathbf{0}}$ are utilized:

$$
\begin{gathered}
{ }^{N} \boldsymbol{v} \\
{ }^{N} \boldsymbol{v}_{0}+{ }^{N} \boldsymbol{a}_{\text {lin }} \cdot \Delta t \\
{ }^{N} \boldsymbol{r} \quad={ }^{N} \boldsymbol{r}_{0}+{ }^{N} \boldsymbol{v}_{0} \cdot \Delta t+\frac{1}{2} \cdot{ }^{N} \boldsymbol{a}_{\text {lin }} \cdot(\Delta t)^{2}
\end{gathered}
$$

Accelerometer and gyroscope sensor errors directly influence the position result. These errors accumulate over time [6]. In Ref. [17] a square trajectory is set as a test scenario applying this simple INS process. The results reported in Ref. [17] show huge errors, which are mainly caused by following factors. First, due to the gyroscope errors, the turning angle at each corner is larger than 90 degrees. Second, the estimated length of each straight path is greater than the real length. This is due to accelerometer errors caused by accelerometer bias in both the stance and swing phase [17]. It is reported that the estimated total travelled distance is ca. $83.5 \mathrm{~m}$, compared to $40 \mathrm{~m}$ real path. Below, methods for alleviating these errors are presented.

\subsubsection{Zero velocity UPdaTe (ZUPT)}

In addition to the errors just stated, there is another source. Due to the noise from the gyroscopes, the computed orientation deviates from the correct orientation. In this way, parts of the gravity component are taking into account as linear acceleration. Due to the errors accumulating over time, huge errors for the position arise $[15,16]$. As can be seen from Fig. 5, the velocity of the foot should be zero, when it touches the ground, i.e. in the stance phase. With the help of ZUPT, the velocity is set to zero at this point of time, alleviating some of the errors. For best results, the IMU should be mounted on the foot. Below the conditions $C_{\text {Stance, } 1}$ to $C_{\text {Stance,3 }}$ for the identification of the stance phase are described according to Ref. [17]:

\section{Magnitude of acceleration}

During the stance phase, the magnitude of the acceleration ${ }^{B} a_{k}$ for each sample $k$ is supposed to be the gravity, i.e. 


$$
C_{\text {Stance }, 1}= \begin{cases}\text { true, } & \text { if } T H_{\text {min }, a c c}<\left.\right|^{B} \boldsymbol{a}_{k} \mid<T H_{\text {max }, a c c} \\ \text { false, } & \text { otherwise }\end{cases}
$$

where

$$
\left.\right|^{B} \boldsymbol{a}_{k} \mid=\sqrt{{ }^{B} a_{x, k}^{2}+{ }^{B} a_{y, k}^{2}+{ }^{B} a_{z, k}^{2}}
$$

Since gravity is contained within the acceleration

$$
\begin{aligned}
T H_{\text {min }, a c c} & :=9.3 \frac{\mathrm{m}}{\mathrm{s}} \\
T H_{\max , a c c} & :=10.3 \frac{\mathrm{m}}{\mathrm{s}}
\end{aligned}
$$

\section{Variance of acceleration}

Moreover, at stance the variance of the acceleration shall be close to zero. Instead of the variance, which is unknown, in Ref. [17] the second sample moment about the sample mean ${ }^{B} M_{k, a c c}^{2}$ is used to form the following condition

$$
C_{\text {Stance }, 2}= \begin{cases}\text { true, } & \text { if }{ }^{B} M_{k, a c c}^{2}<T H_{\max , M_{a c c}^{2}} \\ \text { false, } & \text { otherwise }\end{cases}
$$

The sample moment about the sample mean ${ }^{B} M_{k, a c c}^{2}$ is determined as follows [18]

$$
{ }^{B} M_{k, a c c}^{2}=\frac{1}{2 n+1} \sum_{k-n}^{k+n}\left(||^{B} \boldsymbol{a}_{k} \mid-\overline{||^{B} \boldsymbol{a}_{k} \mid}\right)^{2}
$$

where $\overline{\left|\boldsymbol{a}_{k}\right|}$ is the sample mean for the $2 n+1$ sampling points, i.e.

$$
\overline{\left|\boldsymbol{a}_{k}\right|}=\left.\frac{1}{2 n+1} \sum_{k-n}^{k+n}\right|^{B} \boldsymbol{a}_{k} \mid
$$

In Ref. [17] parameter $n$ is set to 15 . The threshold is defined as

$$
T H_{\max , M_{a c c}^{2}}:=1.5 \frac{\mathrm{m}^{2}}{\mathrm{~s}^{4}}
$$

Eq. (29) shall represent the variance for the sample. Since the mean is not known, we recommend using the sample variance instead, since the expected value of the sampling variance equals the variance of the underlying population [18]. For the sample variance the denominator in Eq. (28) must be changed to 2n.

\section{Magnitude of angular velocity}

The magnitude of the angular velocity ${ }^{B} \boldsymbol{w}_{k}$ is supposed to be zero at stance, i.e.

$$
C_{\text {Stance }, 3}= \begin{cases}\text { true } & \text { if }\left.\right|^{B} \boldsymbol{w}_{k} \mid<T H_{\text {max }, g y r} \\ \text { false } & \text { otherwise }\end{cases}
$$

where

$$
\left|{ }^{B} \boldsymbol{w}_{k}\right|=\sqrt{{ }^{B} w_{x, k}^{2}+{ }^{B} w_{y, k}^{2}+{ }^{B} w_{z, k}^{2}}
$$

The threshold is set to

$$
T H_{\text {max }, g y r}:=1.5 \mathrm{rad} / \mathrm{s}
$$

ZUPT requires the IMU to be attached on the foot. However, in the medical and commercial field, the sensors are often mounted on the ankle, where the velocity at stance is not equal to zero, since the leg is not at rest. Hence, the ZUPT stance detection cannot be working properly. To deal with this, an extension is presented in Section 5, which is called non-ZUPT.

\subsubsection{Summary of INS}

Table 1 is a comparison of different INS techniques. As can be seen, ZUPT can improve the performance significantly. Moreover, an INS based on the non-ZUPT approach is listed. Since ZUPT and non-ZUPT are enhancing the accuracy, we employ both in our PNS.

Table 1 Evaluation of INS algorithms.

\begin{tabular}{llll}
\hline & {$[17]$} & {$[17]$} & {$[32]$} \\
\hline Technique & Basic & ZUPT-aided & Non-ZUPT-aided \\
& & High (2.9\%) & \\
Accuracy & Low (108\%) & $(5.0 \%$ in & High (3.6\%) \\
& & Ref. [21]) & \\
Robustness & High & Medium & Low \\
Performance & Low & Medium & Medium \\
\hline
\end{tabular}

\subsection{PDR}

Another approach for a PNS is PDR. Here, for each step the process consists of three stages: (a) step detection, (b) calculation of the step length and (c) estimation of the orientation. Continuously repeating these steps allows determining the walking trajectory. In contrast to double integration, step recognition only 
generates two-dimensional position results. Below, approaches for step length estimation are summarized.

In Ref. [19] the vertical acceleration is used to estimate the step length $S L_{\text {est }}$ according to

$$
S L_{e s t}=K \cdot \sqrt[4]{a_{v e r t, \max }-a_{v e r t, \text { min }}}
$$

in which $\mathrm{K}$ represents a calibration value for pedestrians and $a_{\text {vert,max }}$ and $a_{\text {vert,min }}$ are the maximum and minimum values of the acceleration in one step.

Another algorithm based on a biomechanical model is described in Refs. [20, 21]. Here the acceleration on the PCOM (Pedestrian's Centre Of Mass) is measured, which is the point at the back near the fourth lumbar vertebra [22]. The estimated step length is calculated as

$$
S L_{e s t}=K \cdot \sqrt{2 l h-h^{2}}
$$

in which $l$ is the leg length of the pedestrian, $K$ is a calibration value and $h$ is the vertical displacement of the PCOM in one step. The PCOM is ascending and $h$ can be described as

$$
h=\int_{T_{0}}^{T_{0}+T_{\text {asc }}}\left(\int_{T_{0}}^{T_{0}+T_{\text {asc }}} a_{\text {vert }, p c o m} d t\right) d t^{\prime}
$$

Here $T_{\text {asc }}$ is the ascending time of the PCOM and $a_{\text {vert,pcom }}$ is the vertical acceleration of the PCOM.

Another empirical algorithm is presented in Ref. [23], where the step frequency $f_{\text {step }}$ and the pedestrian height $h$ are utilized. The estimated step length is as follows

$$
S L_{\text {est }}=h \cdot\left(a \cdot f_{\text {step }}+b\right)+c
$$

where $a, b$ and $c$ are calibration parameters, which are obtained by a complex training process using a short-time Fourier transform.

Table 2 summarizes these algorithms. The high error for the second variant is caused by placing the sensors in the pocket and not on the PCOM.

\section{Orientation Estimation}

Below, multiple techniques are introduced. The Madgwick algorithm, which we employ, is described in detail.

\subsection{Problem Description}

Accurate orientation plays an important role, since incorrect orientation causes parts of the gravitational acceleration to be interpreted as linear acceleration, resulting in huge errors. The problem to estimate the orientation was first formulated by Wahba [24]. There, the DCM $\mathrm{C}_{R}^{B}$ between a reference frame R and a body frame $\mathrm{B}$ is determined by using co-registered vectors. For this, a least-square loss function $L(\cdot)$ is minimized, given as [24, 25].

$$
L\left(\mathrm{C}_{R}^{B}\right)=\frac{1}{2} \cdot \sum_{i}\left(\left.a_{i} \cdot\right|^{B} \boldsymbol{v}_{i}-\left.\mathrm{C}_{R}^{B} \cdot{ }^{R} \boldsymbol{v}_{i}\right|^{2}\right)
$$

Here, ${ }^{B} \boldsymbol{v}_{i}$ is a set of unit vectors measured in the body frame, ${ }^{R} \boldsymbol{v}_{i}$ are the corresponding vectors in the reference frame and the $a_{i}$ denote non-negative weights.

For the solution of this problem, multiple algorithms have been proposed, which can be classified into deterministic and optimal ones [26]. For the deterministic algorithms, the attitude is computed by using a minimal set of measured data and solving non-linear equations. In contrast, the optimal techniques apply more than the minimal set of measured data and minimize a cost function. Below, some algorithms are presented.

The TRIAD algorithm is a deterministic algorithm [27], where two measured non-parallel reference vectors are normalized and combined to construct two triads of orthonormal vectors. In contrast, QUEST is an

Table 2 Evaluation of step length estimation algorithms.

\begin{tabular}{llll}
\hline & Eq. (35) [19] & Eq. (36) [22] & Eq. (38) [23] \\
\hline Technique & Empirical & $\begin{array}{l}\text { Biomechanical } \\
\text { Fourier } \\
\text { transform }\end{array}$ \\
Accuracy & $6.3 \%$ & $\begin{array}{l}20.5 \% \\
(5.0 \% \text { in Ref. }\end{array}$ & $2.5 \%$ \\
& & $21])$ & \\
Robustness & High & Medium & Low \\
$\begin{array}{l}\text { Calibration } \\
\text { efforts }\end{array}$ & Simple & Medium & High \\
Performance & Medium & Medium & High \\
\hline
\end{tabular}


optimal algorithm where Davenport's q-method is applied to find the optimal quaternion which is a transformation of Wahba's loss function [26, 27]. In addition, there are many KF (Kalman Filter) based approaches. Here, accelerometer and magnetometer data are fused to alleviate the drift from the gyroscopes. As an example, a quaternion based EKF (Extended Kalman Filter) is presented in Ref. [28]. An alternative to the KF is the CF (Complementary Filter). Compared to $\mathrm{KF}$, it is simpler and more effective. Examples in Refs. [29, 30] present that the human motion tracking using EKF needs to be captured at sampling rates above $512 \mathrm{~Hz}$, which exceeds the maximum sampling rate of $200 \mathrm{~Hz}$ of Android-based devices to be used in this paper. In Ref. [7], the Madgwick algorithm is presented, which will be used in our system. It is a highly effective CF, utilizing the gradient descent technique. It employs accelerometer and magnetometer data to alleviate the drift of the gyroscopes. It is presented below.

\subsection{Madgwick Algorithm [7]}

Let ${ }^{N} \hat{\boldsymbol{G}}$ denote the normalized vector of the gravitational field in the local navigation frame and let ${ }^{B} \hat{\boldsymbol{g}}$ be the associated measured field in the body frame. Since gravitation is directed into the earth, ${ }^{N} \hat{\boldsymbol{G}}$ only exhibits one vertical component, i.e.

$$
{ }^{N} \hat{\boldsymbol{G}}=[0,0,1]^{T}
$$

According to Eq. (18), ${ }^{N} \hat{\boldsymbol{G}}$ can be transformed into the body frame. Hence, the difference $f_{g}$ between the field, transformed into the body frame, and the measured field can be written as

$$
f_{g}=\left(\hat{\mathfrak{q}}_{N \leftarrow B}^{*} \otimes{ }^{N} \hat{\mathfrak{G}}_{p} \otimes \hat{\mathfrak{q}}_{N \leftarrow B}\right)-{ }^{N} \hat{\mathfrak{g}}_{p}
$$

where ${ }^{N} \hat{\mathfrak{G}}_{\mathfrak{p}}$ and ${ }^{N} \hat{\mathfrak{g}}_{\mathfrak{p}}$ are the pure quaternions of ${ }^{N} \hat{\boldsymbol{G}}$ and ${ }^{B} \hat{\boldsymbol{g}}$. Analogous, the difference $f_{m}$ between the magnetic field ${ }^{N} \hat{\boldsymbol{M}}$ and the measured counterpart ${ }^{B} \hat{\boldsymbol{m}}$ in the body frame can be written as

$$
f_{m}=\left(\hat{\mathfrak{q}}_{N \leftarrow B}^{*} \otimes{ }^{N} \hat{\mathfrak{M}}_{p} \otimes \hat{\mathfrak{q}}_{N \leftarrow B}\right)-{ }^{N} \hat{\mathfrak{m}}_{p}
$$

In contrast to ${ }^{N} \hat{\boldsymbol{G}}$, the earth's magnetic field ${ }^{N} \hat{\boldsymbol{M}}$ has one component along the north direction. It has no component along the east direction. Moreover, due to the curvature of the earth, the orientation of the magnetic field can be directed into the earth, hence there is also a component along the vertical direction. The inclination specifies this angle between the horizontal and this field. It is approximately $65^{\circ}$ in Dresden, Germany [31]. Hence,

$$
{ }^{N} \hat{\boldsymbol{M}}=\left[M_{\text {north }}, 0, M_{\text {vertical }}\right]^{T}
$$

Additionally, there is a deviation between the magnetic north and the true north. This angle, denoted as declination, between lines from the observer to magnetic and true north is small for locations far from the North Pole. During the Madgwick algorithm we assume the declination to be $0^{\circ}$.

Note, that $f_{g}$ and $f_{m}$ are functions of $\hat{\mathfrak{q}}_{N \leftarrow B}$. Depending on the availability of the sensors, either $f_{g}$ or $f_{m}$ or both should be minimized to determine the orientation. To encompass all cases we define $f$ as

$$
f= \begin{cases}{\left[f_{g}\right],} & \text { incorporating gravitational } \\
{\left[\begin{array}{l}
f_{g} \\
f_{m}
\end{array}\right],} & \text { incorporating gravitational } \\
\text { and magnetic field (GMF) }\end{cases}
$$

and our object is to minimize $f$. According to Ref. [7], the gradient descent algorithm is applied, which is iteratively performed:

$$
\mathfrak{q}_{N \leftarrow B_{k}}=\hat{\mathfrak{q}}_{N \leftarrow B_{k-1}}-\mu \frac{\nabla F_{k}}{\left\|\nabla F_{k}\right\|}, \text { Start }: \hat{\mathfrak{q}}_{N \leftarrow B_{0}}
$$

Here, $\mu$ is the step size, $\hat{\mathfrak{q}}_{N \leftarrow B_{0}}$ is the initial orientation, which could be determined with the help of a magnetometer. $f_{k}$ represents the value of $f$ in each iteration, i.e.

$$
f_{k}=\left\{\begin{array}{l}
{\left[\left(\hat{\mathfrak{q}}_{N \leftarrow B_{k-1}}^{*} \otimes \hat{\mathfrak{G}}_{p} \otimes \hat{\mathfrak{q}}_{N \leftarrow B_{k-1}}\right)-^{N} \hat{\mathfrak{g}}_{p}\right], \quad G F} \\
{\left[\begin{array}{l}
\left(\hat{\mathfrak{q}}_{N \leftarrow B_{k-1}}^{*} \otimes \hat{\mathfrak{G}}_{p} \otimes \hat{\mathfrak{q}}_{N \leftarrow B_{k-1}}\right)-^{N} \hat{\mathfrak{g}}_{p} \\
\left(\hat{\mathfrak{q}}_{N \leftarrow B_{k-1}}^{*} \otimes \hat{\mathfrak{M}}_{p} \otimes \hat{\mathfrak{q}}_{N \leftarrow B_{k-1}}\right)-^{N} \hat{\mathfrak{m}}_{p}
\end{array}\right], G M F}
\end{array}\right.
$$

Instead of minimizing $f$ or $f_{k}$ directly, the 
minimization of the squared error loss $F_{k}$ is employed which is written as

$$
F_{k}=\frac{1}{2} f_{k}^{T} \circ f_{k}
$$

Applying the chain rule, it can be easily shown that

$$
\nabla F_{k}=J_{f_{k}}^{T} \circ f_{k}
$$

where $J_{f_{k}}$ is the Jacobian of $f_{k}$.

The application of Eq. (45) is the first part of the Madgwick algorithm. In parallel, the orientation determined with the help of the measured angular velocity from the gyroscope is available (see Eq. (20)). The application of Eqs. (20) and (21) for time step $k$ reads

$$
\mathfrak{q}_{N \leftarrow B, \omega} \approx \hat{\mathfrak{q}}_{N \leftarrow B_{k-1, e s t}}+\dot{\mathfrak{q}}_{N \leftarrow B_{k, \omega}} \cdot \Delta t
$$

where

$$
\dot{\mathfrak{q}}_{N \leftarrow B_{k, \omega}}=\frac{1}{2} \cdot \hat{\mathfrak{q}}_{N \leftarrow B_{k-1, e s t}} \otimes^{B} \mathfrak{w}_{\mathfrak{p}}
$$

Here, we have used the subscript $\omega$ to distinguish the orientation determined with the help of the angular velocity from the orientation determined by the gradient descent algorithm of Eq. (45). For the latter, we will use the subscript $\nabla$ in the remainder of this paper. Both orientations are combined by means of a $\mathrm{CF}$ to determine the final orientation estimator:

$$
\mathfrak{q}_{N \leftarrow B_{k, e s t}}=\gamma \cdot \mathfrak{q}_{N \leftarrow B_{k, \nabla}}+(1-\gamma) \cdot \mathfrak{q}_{N \leftarrow B_{k, \omega}}, 0 \leq \gamma \leq 1
$$

We use the index est to specify this final orientation estimate. $\gamma$ is the weight of the filter. Generally, the application of Eq. (45) requires multiple iteration steps before applying the CF in Eq. (51). According to Ref. [7], one step is sufficient, if the convergence rate is greater than or equal to the rate of change of the physical orientation, which can be realized by selecting an appropriate value of the step size $\mu$. Below, this case is considered. In doing so, the individual iteration steps of Eq. (45) can be thought of being timely aligned to the measured angular velocity. As a modification to Eq. (45), the CF result $\mathfrak{q}_{N \leftarrow B_{k, \text { est }}}$ is therefore used directly in place of $\hat{\mathfrak{q}}_{N \leftarrow B_{k}}$ :

$$
\mathfrak{q}_{N \leftarrow B_{k, V}}=\hat{\mathfrak{q}}_{N \leftarrow B_{k-1, e s t}}-\mu \frac{\nabla F_{k}}{\left\|\nabla F_{k}\right\|}
$$

Measurements of the magnetic field in the vicinity of electrical circuits or metal structures can lead to significant errors in the inclination. These errors can be made visible by back-propagating the measured magnetic field in the body frame into the local navigation frame $\mathrm{N}$, according to Eq. (17), i.e.

$$
{ }^{N} \hat{\mathfrak{n}}_{p, k}:=\hat{\mathfrak{q}}_{N \leftarrow B_{k-1, e s t}} \otimes{ }^{B} \hat{\mathfrak{m}}_{p, k} \otimes \hat{\mathfrak{q}}_{N \leftarrow B_{k-1, \text { est }}}^{*}
$$

Ideally, the direction of ${ }^{N} \hat{\mathfrak{M}}_{p}$ and ${ }^{N} \hat{\mathfrak{n}}_{k}$ should match for every time step $k$. However, magnetic distortion produces inclination errors, which are visible as non-zero components in east direction, i.e.

$$
{ }^{N} \hat{\mathfrak{n}}_{p, k}=\left[0, N_{\text {north }, k}, N_{\text {east }, k}, N_{\text {vert }, k}\right]^{T}
$$

These effects of erroneous inclination can be reduced by simply rotating the magnetic field vector about the $\mathrm{z}$-axis in such a way, that the east component vanishes. This quaternion

$$
{ }^{N} \hat{\mathfrak{O}}_{p, k}:=\left[0, \sqrt{N_{\text {north }, k}^{2}+N_{\text {east }, k}^{2}}, 0, N_{\text {vert }, k}\right]^{T}
$$

can then be used in place of ${ }^{N} \hat{\mathfrak{M}}_{p}$ within the next iteration, e.g. in Eq. (46).

Table 3 broadly summarizes the aforementioned orientation estimation approaches.

Table 3 Evaluation of orientation estimation algorithms

\begin{tabular}{lllll}
\hline & TRIAD & QUEST & EKF [28] & CF[7] \\
\hline $\begin{array}{l}\text { Magnetic } \\
\text { perturbation }\end{array}$ & Sensitive & Sensitive & Median & Median \\
$\begin{array}{l}\text { Implementation } \\
\text { efforts }\end{array}$ & Simple & Simple & Median & Median \\
$\begin{array}{l}\text { Orientation } \\
\text { error } \\
\begin{array}{l}\text { Convergence } \\
\text { speed }\end{array}\end{array}$ & Large & Large & Small & Small \\
\hline
\end{tabular}

\section{PNS Structure}

To benefit from the advantages of both the INS and the PDR, our system consists of two sub-systems as shown in Fig. 9. For the INS, a smartwatch is employed, which is attached to the ankle. In contrast, a 
smartphone is used for the PDR to be held at hand. Both subsystems will be detailed in the subsequent paragraphs. This is followed by the description of the fusion process for the final output.

\subsection{INS}

Below, the general form of our INS is illustrated. Afterwards, methods for improving the accuracy are described.

\subsubsection{INS Structure}

Fig. 10 shows the structure for our INS, which is mostly equivalent to Fig. 8. Likewise, the underlying mathematics is mainly identical to the ones in Section 3.1. The only modification is due to the Madgwick algorithm, which requires accelerometer data in addition to gyroscope measurements to estimate the orientation. However, we have modified the Madgwick algorithm, to cope with magnetic disturbances. The details are given below in Section 5.1.2.

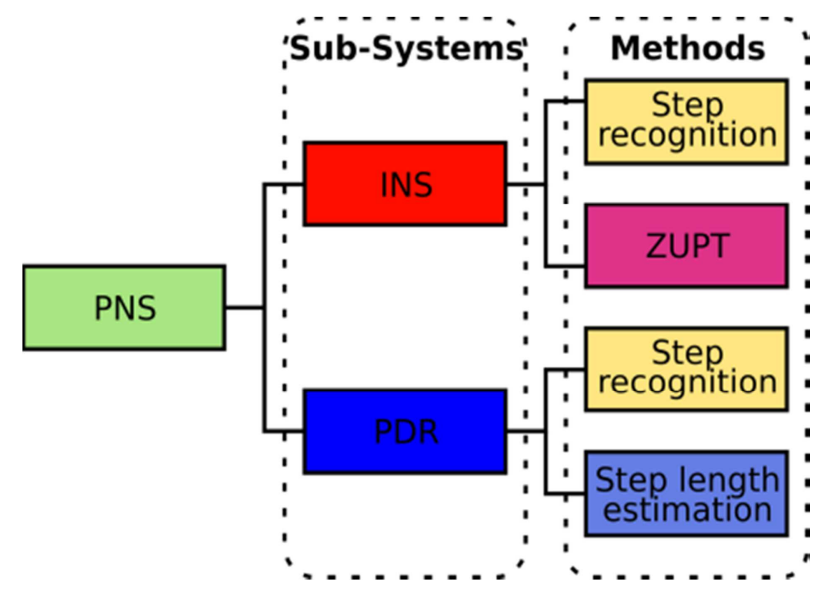

Fig. 9 System structure.

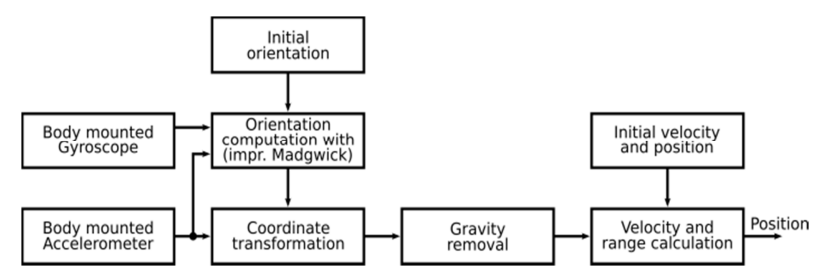

Fig. 10 Flowchart of INS in our PNS.

After performing the improved Madgwick algorithm at time $k$, the measured acceleration from the same time, characterized as a pure quaternion ${ }^{B} \mathfrak{a}_{\mathfrak{p}, \mathrm{k}}$, is first transformed from the body frame into the $N$ frame according to Eq. (56). The vector part of the result ${ }^{N} \mathfrak{a}_{\mathfrak{p}, \mathrm{k}}$ specifies the acceleration vector in the $N$ frame, denoted as ${ }^{N} \boldsymbol{a}_{k}$. Afterwards, gravity is removed as shown in Eq. (57). The velocity and the range can then be determined with the help of Eqs. (58) and (59).

$$
\begin{gathered}
{ }^{N} \mathfrak{a}_{\mathfrak{p}, \mathrm{k}}=\hat{\mathfrak{q}}_{N \leftarrow B_{k}} \otimes{ }^{B} \mathfrak{a}_{\mathfrak{p}, \mathrm{k}} \otimes \hat{\mathfrak{q}}_{N \leftarrow B_{k}}^{*} \\
{ }^{N} \boldsymbol{a}_{l i n, k}={ }^{N} \boldsymbol{a}_{k}-{ }^{N} \boldsymbol{G} \\
{ }^{N} \boldsymbol{v}_{k}={ }^{N} \boldsymbol{v}_{k-1}+{ }^{N} \boldsymbol{a}_{l i n, k} \cdot \Delta t \\
\boldsymbol{r}_{k}={ }^{N} \boldsymbol{r}_{k-1}+{ }^{N} \boldsymbol{v}_{k-1} \cdot \Delta t+\frac{1}{2} \cdot{ }^{N} \boldsymbol{a}_{l i n, k} \cdot(\Delta t)^{2}
\end{gathered}
$$

\subsubsection{Upgrades}

For improving the accuracy, we employ two techniques, i.e. the ZUPT method from Section 3.1 and another approach, denoted as non-ZUPT. Both are described in turn. However, firstly an enhanced version for detecting the stance phase is presented, which is the basis for both methods.

\section{Stance Phase Detection}

Fig. 11 shows the conditions for detecting the stance phase. $C_{\text {Stance, } 1}$ to $C_{\text {Stance,3 }}$ (according to Ref. [17]) have already been described. However, for increasing the precision, we have added conditions $C_{\text {Stance, } 4}$ and $C_{\text {Stance,5 }}$, as we will show in our measurements. All conditions need to be fulfilled simultaneously, which is characterized by the logical AND.

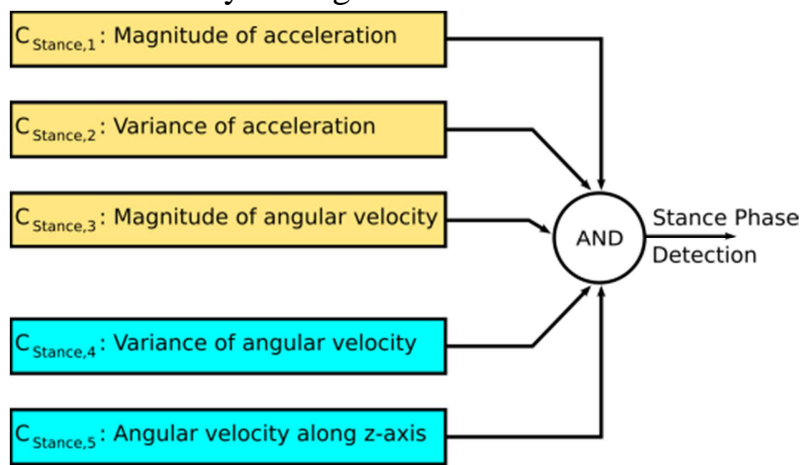

Fig. 11 Logic for detecting the stance phase.

$C_{\text {Stance,4 }}$ shall express that at stance the variance of the angular velocity is smaller than a threshold $T H_{\max , M_{g y r}^{2}}$. Instead of the variance, which is unknown, the sample moment about the sample mean ${ }^{B} M_{k, g y r}^{2}$ is 
employed. Thus, $C_{\text {Stance, } 4}$ reads

$$
C_{\text {Stance } 4}= \begin{cases}\text { true, } & \text { if }{ }^{B} M_{k, g y r}^{2}<T H_{\max , M_{g y r}^{2}} \\ \text { false, } & \text { otherwise }\end{cases}
$$

where ${ }^{B} M_{k, g y r}^{2}$ is determined as Ref. [18]

$$
{ }^{B} M_{k, g y r}^{2}=\frac{1}{2 n+1} \sum_{k-n}^{k+n}\left(\left|\omega^{B} \omega_{k}\right| \overline{-\omega_{k}^{B} \mid}\right)^{2}
$$

Here, $\overline{\left.\right|^{B} \omega_{k} \mid}$ is the sample mean for the $2 n+1$ sampling points:

$$
\overline{\mid \omega_{k}}\left|=\frac{1}{2 n+1} \sum_{k-n}^{k+n}\right|^{B} \omega_{k} \mid
$$

In most commercial and clinical applications, the IMU is preferred to be mounted on the ankle instead of on the foot [32]. Therefore, we also have mounted the IMU on the ankle in our experiments, as shown in Fig. 12. As can be seen, the body frame is aligned such, that the $\mathrm{x}$-axis points upward, the $\mathrm{y}$-axis points in walking direction and the $\mathrm{z}$-axis points in the direction of the viewer. At stance, the z-component of the angular velocity shall be zero (see Fig. 13) [33]. Therefore, we have added a condition in this regard, whose necessity becomes obvious in the experiments.

$$
C_{\text {Stance }, 5}= \begin{cases}\text { true, } & \text { if }\left.\right|^{B} \omega_{z, k} \mid<T H_{\max , g y r, z} \\ \text { false, } & \text { otherwise }\end{cases}
$$

Here, ${ }^{B} \omega_{z, k}$ is the z-value of ${ }^{B} \omega_{k}$ from sample k, and $T H_{\max , g y r, z}$ is a threshold to be defined.

\section{ZUPT and non-ZUPT method}

As explained in Section 2.4, the swing phase occupies around $40 \%$ of the full gait-cycle for continuous walking. This time can be measured and is the criteria for detecting if a stride is not successive with the previous one, which means that continuous walking is interrupted. Therefore, we set the condition

$$
C_{\text {Successive }}= \begin{cases}\text { true, } & \text { if } T_{\text {Swing }} \geq 2 / 3 \cdot T_{\text {Stance }} \\ \text { false, } & \text { otherwise }\end{cases}
$$

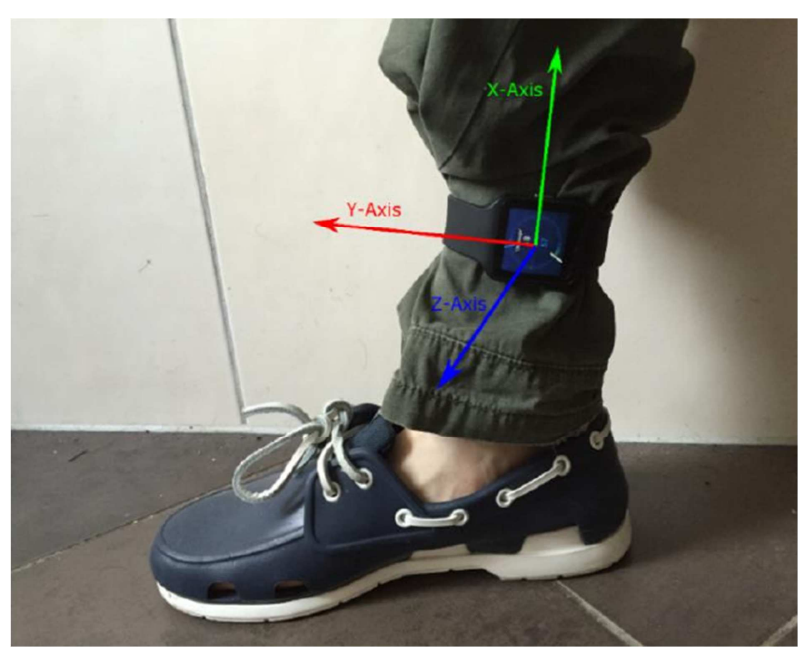

Fig. 12 IMU attached to the ankle.

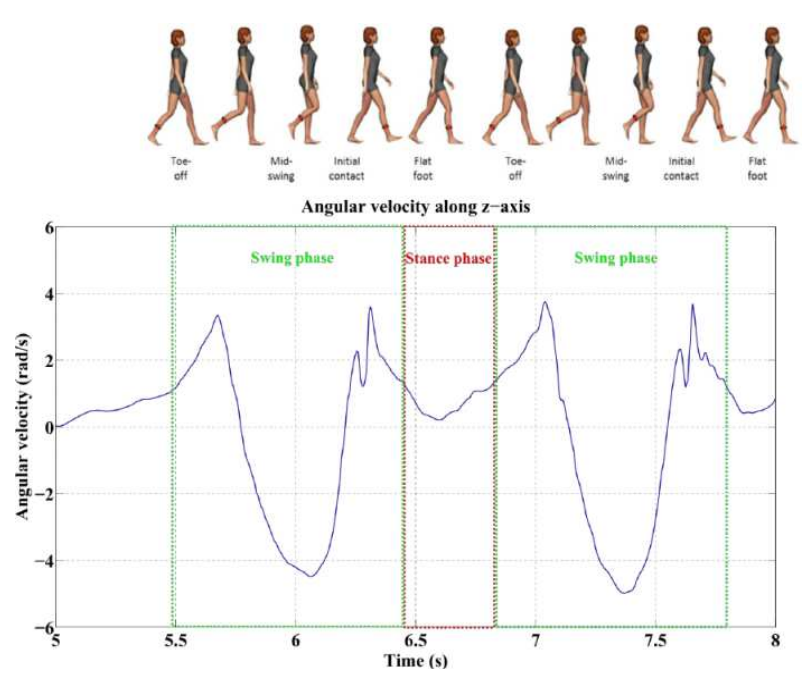

Fig. 13 Angular velocity along the z-axis ([33] modified).

The factor $2 / 3$ arises, if one firstly determines the expected time for a full gait-cycle $T_{\text {Gait exp }}$ for normal continuous walking, i.e. for assuming successive strides:

$$
T_{\text {Gait }, \text { exp }}:=\frac{100}{60} \cdot T_{\text {Stance }}
$$

Thus, the expected duration of the swing phase $T_{\text {Swing,exp }}$ is

$$
T_{\text {Swing,exp }}:=0.40 \cdot T_{\text {Gait }, \text { exp }}=\frac{40}{60} \cdot T_{\text {Stance }}=\frac{2}{3} \cdot T_{\text {Stance }}
$$

If the duration of the swing phase $T_{\text {Swing }}$ is below $T_{\text {Swing,exp }}$, then walking is considered non-successive, since a long stance phase indicates that the person is standing. In this case, we apply the ZUPT method; otherwise we use non-ZUPT: 
Technique $= \begin{cases}\text { non-ZUPT, } & \text { if } C_{\text {Successive }}=\text { true } \\ \text { ZUPT, } & \text { otherwise }\end{cases}$

The reason for this is that the velocity should only be set to zero, according to ZUPT, if the person is fully standing, i.e. if the strides are not successive. Would the IMU being attached directly on the foot, then ZUPT could be used for every stride. However for the IMU being placed at the ankle, the velocity will not go back to zero, since the ankle can be in motion even if the velocity of the foot is zero. Hence ZUPT cannot be used in this case. Instead, we apply the non-ZUPT technique, introduced in Ref. [32]. It is presented below.

In contrast to ZUPT, the initial velocity is not set to zero, but is calculated with the help of Fig. 14. When the pedestrian is at mid-stance (please see Fig. 5), there exists an angular velocity ${ }^{B} \omega_{t}$ approximately tangential to the ground surface. Referred to Fig. 12, it points in the direction of the z-axis. The velocity of the IMU in the body frame can then be computed as:

$$
{ }^{B} \boldsymbol{v}_{t}={ }^{B} \boldsymbol{\omega}_{t} \times{ }^{B} \boldsymbol{d}
$$

This means the tangential velocity ${ }^{B} \boldsymbol{v}_{t}$ of the IMU is the cross product of the angular velocity and the rotation moment arm ${ }^{B} \boldsymbol{d}$, which points from the ground to the IMU. ${ }^{B} \boldsymbol{v}_{t}$ is then transformed to ${ }^{N} \boldsymbol{v}_{t}$ according to Eq. (17).

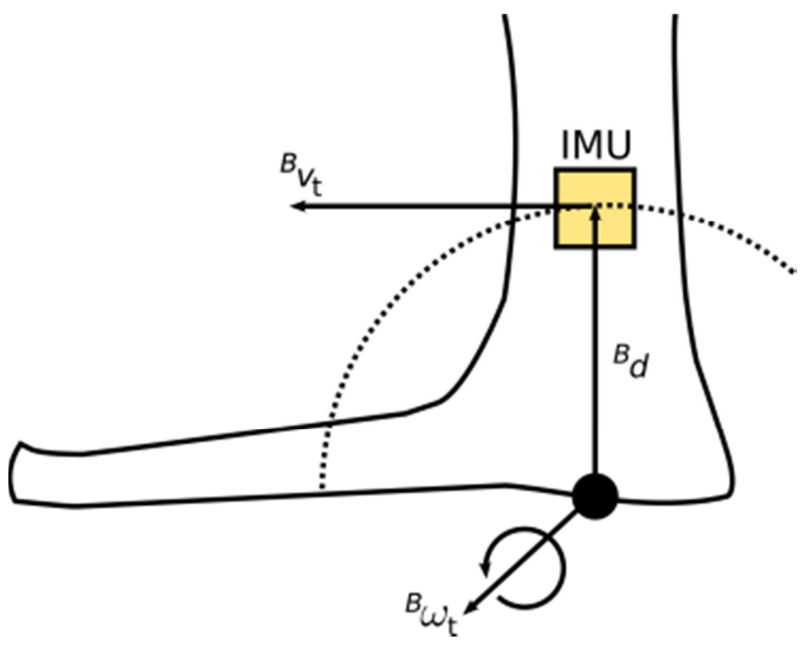

Fig. 14 Tangential velocity at mid-stance.

\section{Orientation Estimation}

In this paragraph some updates to the basic Madgwick algorithm are presented, which we employ in our PNS. As explained in Section 4.2, the accelerometers should only measure the gravitational acceleration and the magnetometers should only measure the earth's magnetic field. Hence, the basic Madgwick algorithm assumes that linear accelerations, e.g. due to motion, and magnetic distortions can be neglected. In particular the first assumption is not valid, especially for the gait of a pedestrian in the swing phase. As shown in Ref. [34], the linear acceleration can reach up to $5 \mathrm{~m} / \mathrm{s}$. Moreover, temporary high-intensive magnetic distortion also affects the performance. Inspired by Ref. [35], we therefore have added some conditions for the usage of the algorithm. Firstly, the Madgwick algorithm is only applied at stance, where the linear acceleration should be at a minimum, otherwise the gyroscope data are used for determining the orientation:

$$
C_{\text {Orientation }}= \begin{cases}C_{\text {Madgwick }}, & \text { if stance phase } \\ \text { Gyroscope, }, & \text { otherwise }\end{cases}
$$

Furthermore, if the magnitude of the measured magnetic field ||$^{B} \boldsymbol{m} \mid$ and the magnitude of the reference earth's magnetic field $\left.\right|^{N} \boldsymbol{b}_{\text {ref }} \mid$ (calculated by the World Magnetic Model [31]) are greater than a threshold $T H_{\text {mag }}$, the magnetic field is neglected because of present magnetic distortion. This condition is stated below, where a typical value is given as $T H_{m a g}=30 \mu T$.

$$
C_{\text {Madgwick }}=\left\{\begin{array}{l}
\mathrm{GF}, \quad \text { if }\left\|^{B} \boldsymbol{m}|-|^{N} \boldsymbol{b}_{\text {ref }}\right\|>T H_{\text {mag }} \\
\mathrm{GMF}, \text { otherwise }
\end{array}\right.
$$

Here, the abbreviations GF and GMF from Eq. (44) were used. The full process for determining the orientation is illustrated in Fig. 15. The respective equation numbers are listed under each block. 
Diamond symbols specify selections according to Eqs. (67) or (68). $\hat{\mathfrak{q}}_{N \leftarrow B_{k, \text { st }}}$ is the final output at time step $\mathrm{k}$. Dotted lines designate variables which become valid in the next time step $\mathrm{k}+1$.

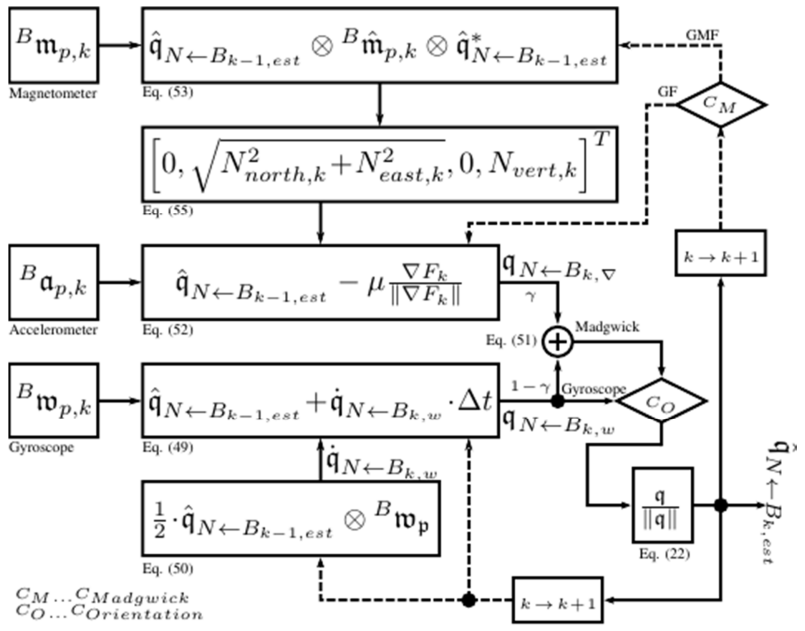

Fig. 15 Process of the Madgwick algorithm ([7] modified).

\subsection{PDR}

In our PNS the IMU for the INS is attached on the ankle. In addition, another IMU is utilized for the PDR. This IMU constitutes a smartphone which the user holds in his hands. The main error source for an INS is small measurement errors which are accumulated over time. One way to alleviate these errors is by using ZUPT and non-ZUPT. Another alternative, described below, is to apply a PDR system in parallel, to limit the errors.

Different PDR algorithms were described in Section 3.2 , especially in Table 2. For our PNS, we have selected the Weinberg algorithm [19]. Although Renaudin's algorithm [23] is more precise, the robustness of Weinberg's algorithm is higher and the calibration process is easier. Besides, in Ref. [36] it is shown that the Weinberg's algorithm performs best, if the IMU is mounted on the waist or held in the hand steadily.

To determine the vertical acceleration, required in Eq. (35), firstly the acceleration ${ }^{N} \mathfrak{a}_{p, k}$ at point $k$ in the $\mathrm{N}$-frame is calculated according to Eq. (17).

$$
{ }^{N} \mathfrak{a}_{\mathfrak{p}, \mathrm{k}}=\hat{\mathfrak{q}}_{N \leftarrow B_{k}} \otimes{ }^{B} \mathfrak{a}_{\mathfrak{p}, \mathrm{k}} \otimes \hat{\mathfrak{q}}_{N \leftarrow B_{k}}^{*}
$$

where $\hat{\mathfrak{q}}_{N \leftarrow B_{k}}$ is the result from the Madgwick algorithm. The vertical acceleration is then simply the fourth component of this quaternion. The remaining constant $K$ in Eq. (35) is obtained individually for each user via a calibration process, e.g. by walking a distance of $5 \mathrm{~m}$. Fig. 16 shows the vertical acceleration for a walking sequence. The strides are detected with the help of the techniques described in Section 5.1.2 and are separated by red lines.

\subsection{Fusion of INS and PDR}

Table 4 compares the characteristics of the PDR and INS. Since the update rate of the PDR system is low and the technique for calculating the step length is simple, the accuracy is worst for the PDR. However, it can be used complementary to the ZUPT/non-ZUPT INS. Due to the good performance of the INS, the PDR

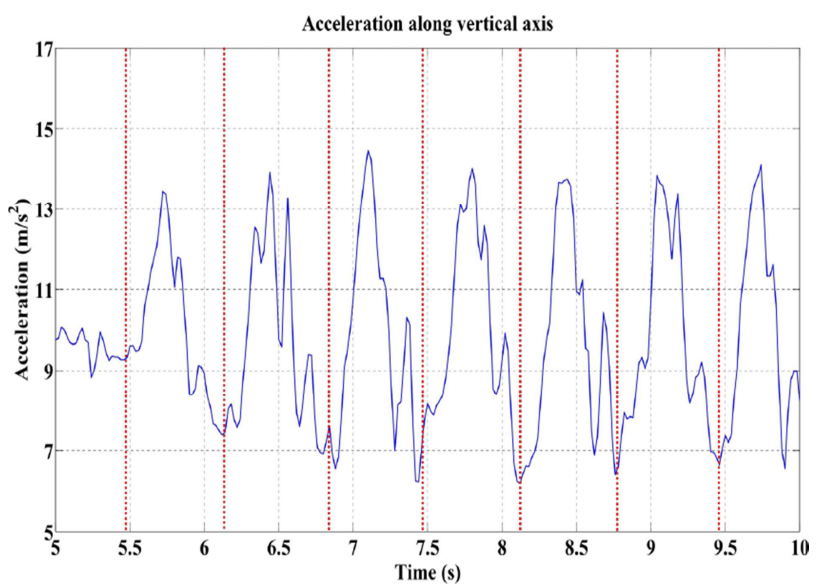

Fig. 16 Measured magnitude of vertical acceleration in a walking sequence.

Table 4 Comparison of PDR and INS.

\begin{tabular}{|c|c|c|}
\hline Characteristics & PDR & INS \\
\hline Procedure & $\begin{array}{l}\text { Simple } \\
\text { (stride length) }\end{array}$ & $\begin{array}{l}\text { Complex } \\
\text { (double integration) }\end{array}$ \\
\hline Accuracy & $\begin{array}{l}\text { Medium } \\
\text { (Requirement: } \\
\text { accurate step } \\
\text { detection and } \\
\text { orientation }\end{array}$ & $\begin{array}{l}\text { High } \\
\text { (Requirement: } \\
\text { accurate stance-phase } \\
\text { detection and } \\
\text { orientation) }\end{array}$ \\
\hline Usage & Flat (2D) & Terrain (3D) \\
\hline Placement & $\begin{array}{l}\text { Head, waist, } \\
\text { hand-held }\end{array}$ & Foot, ankle, leg \\
\hline Sensor quality & Low & High \\
\hline Update rate & $\begin{array}{l}\text { Low } \\
\text { (update when step } \\
\text { detected) }\end{array}$ & $\begin{array}{l}\text { High } \\
\text { (sampling rate of } \\
\text { sensors) }\end{array}$ \\
\hline
\end{tabular}


system is applied simply to modify the stride length calculated by the INS. Therefore, we are employing another complementary filter, i.e.

$$
{ }^{N} \boldsymbol{r}_{k, e s t}=\alpha \cdot{ }^{N} \boldsymbol{r}_{k, I N S}+(1-\alpha) \cdot{ }^{N} \boldsymbol{r}_{k, P D R}, \quad 0 \leq \alpha \leq 1
$$

where ${ }^{N} \boldsymbol{r}_{k, e s t}$ is the estimated length of the k-th stride and ${ }^{N} \boldsymbol{r}_{k, I N S}$ or ${ }^{N} \boldsymbol{r}_{k, P D R}$ is the estimated length of the stride by the INS or PDR. Again, $\alpha$ is the filter weight.

Fig. 17 illustrates the full process. In accordance to Table 4, we apply for the INS the IMU of the smartwatch, which is attached on the ankle, and for the PDR the IMU of the smartphone, which is held in the hand. The fusion takes place in real-time and is performed in the smartphone. For this reason a permanent Bluetooth data connection between both entities is maintained.

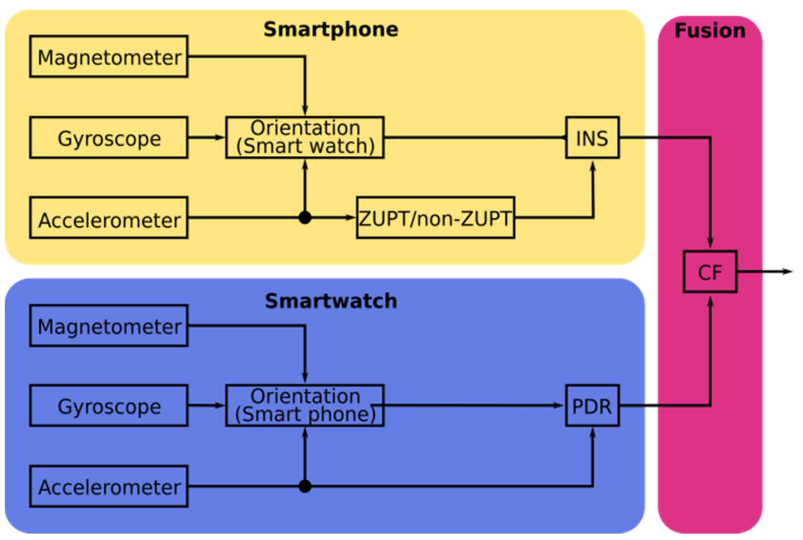

Fig. 17 PNS process.

\section{Experiments}

In this section the set-up of the experiments is introduced firstly. Afterwards, the results are presented.

\subsection{Experimental Set-up}

After presenting the hardware and software utilized, details regarding sampling times, configuration, error metric and scenarios are described.

\subsubsection{Hardware and Software}

For our measurements, we employ the Sony SmartWatch 3 together with the HTC One M8 smartphone. Tables 5 and 6 detail the specifications of both entities. The access to the sensors as well as the communication between both IMUs is performed with the help of the API (application programming interface). Finally, the evaluation of the sensory data is performed in MATLAB for the experiments, whereas it would be implemented on the smartphone for a real user application.

The sensors are accessed via the Android API. There are two types of sensors: raw sensors and synthetic (or virtual) sensors. Raw sensors directly provide the outputs of the MEMS sensors, whereas the synthetic sensors perform some form of preprocessing, filtering or fusion before returning the data. For our PNS we employ raw sensors only.

Table 5 Specification of the Sony SmartWatch 3.

\begin{tabular}{|l|l|}
\hline Operating System & Android Wear 6.0.1 \\
\hline Google Play Services & Version 9.4.52 \\
\hline Processor & $\begin{array}{l}\text { Quad-core ARM Cortex A7 1.2GHz } \\
\text { CPU }\end{array}$ \\
\hline RAM & $512 \mathrm{MB}$ \\
\hline Radios & $802.11 \mathrm{a} / \mathrm{b} / \mathrm{g} / \mathrm{n} / \mathrm{ac}$ Wi-Fi, Bluetooth 4.1 \\
\hline Sensors & $\begin{array}{l}\text { Accelerometer, magnetometer, } \\
\text { gyroscope }\end{array}$ \\
\hline Dimensions & $36 \mathrm{~mm} \times 10 \mathrm{~mm} \times 51 \mathrm{~mm}(74$ grams $)$ \\
\hline
\end{tabular}

Table 6 Specification of the HTC One M8.

\begin{tabular}{|c|c|}
\hline Operating System & Android Wear 6.0 \\
\hline Google Play Services & Version 9.4 .52 \\
\hline Processor & $\begin{array}{l}\text { Qualcomm MSM8974AB Snapdragon } \\
801\end{array}$ \\
\hline RAM & $2 \mathrm{~GB}$ \\
\hline Radios & $802.11 \mathrm{a} / \mathrm{b} / \mathrm{g} / \mathrm{n} / \mathrm{ac}$ Wi-Fi, Bluetooth 4.1 \\
\hline Sensors & $\begin{array}{l}\text { Accelerometer, magnetometer, } \\
\text { gyroscope }\end{array}$ \\
\hline Dimensions & $\begin{array}{l}146.4 \mathrm{~mm} \times 70.6 \mathrm{~mm} \times 9.4 \mathrm{~mm}(160 \\
\text { grams })\end{array}$ \\
\hline
\end{tabular}

\subsubsection{Alignment of Sampling Times}

We have configured the IMUs to have a maximum sampling frequency of $200 \mathrm{~Hz}$. Unfortunately, the sampling times of the accelerometer, gyroscope and magnetometer are not synchronized. Hence, to timely align these samples, we apply a linear interpolation for the raw output data of the sensors. This enables to synchronize the sensors within a single IMU. Nevertheless, we are employing two IMUs, i.e. in the 
smartphone and in the smartwatch. Thus, synchronization between both is required. We solve this issue by performing a calibration prior to the measurements. In our experiments, the user is requested to jump twice at the beginning. This motion is recognized by both IMU and enables to synchronize the time.

\subsubsection{Configuration}

Table 7 summarizes the parameters being used in our experiments. These were determined with the help of preliminary tests. We set $n=33$, thus $2 n+1=67$ points are considered for calculating the variance of the acceleration and angular velocity. For our sampling frequency of $200 \mathrm{~Hz}$ this is approximately $30 \%$ of the time period of one stride. The other parameters were configured with the objective to avoid incorrect stance phase detections.

To circumvent PDR outliers, we have included a separate condition for $\alpha$. This will be described in more detail in Section 6.2.3.

\subsubsection{Error Metric}

To quantify our results, we measure the error relative to the TTD (Total Travelled Distance) $D_{T T D}[15]$. Hence, our relative error $e_{T T D}$ is defined as

$$
e_{T T D}=\frac{\Delta r}{D_{T T D}}
$$

where $\Delta r$ is the absolute position error for the end point of the walking trajectory in the local navigation frame. Utilizing $\Delta r_{\text {north }}$ and $\Delta r_{\text {east }}$ as differences in north and east direction, for two-dimensional considerations $\Delta r$ reads

$$
\Delta r=\sqrt{\left(\Delta r_{\text {north }}\right)^{2}+\left(\Delta r_{\text {east }}\right)^{2}}
$$

\subsubsection{Scenarios}

We perform five different types of experiments, as listed in Table 8. These are specified in more detail in the next subsection.
Table 7 Constants definitions.

\begin{tabular}{ll}
\hline Constant & Value \\
\hline$n$ & 33 \\
$K$ & 0.49 \\
$T H_{\min , a c c}$ & $9.3 \mathrm{~m} / \mathrm{s}$ \\
$T H_{\max , a c c}$ & $10.3 \mathrm{~m} / \mathrm{s}$ \\
$T H_{\max , M_{a c c}^{2}}$ & $1.5 \mathrm{~m}^{2} / \mathrm{s}$ \\
$T H_{\max , g y r}$ & $1.5 \mathrm{rad} / \mathrm{s}$ \\
$T H_{\text {max }, M_{g y r}^{2}}$ & $0.6 \mathrm{rad} / \mathrm{s}^{2}$ \\
$T H_{\max , g y r, z}$ & $1.0 \mathrm{rad} / \mathrm{s}$ \\
$\alpha$ & $\left\{\begin{array}{l}0.5, \text { if } 0.8<\frac{||^{N} \boldsymbol{r}_{k, I N S} \mid}{\left.\right|^{N} \boldsymbol{r}_{k, P D R} \mid}<1.2 \\
1.0, \quad \text { otherwise }\end{array}\right.$ \\
\hline
\end{tabular}

\begin{tabular}{|c|c|}
\hline Type & Specification \\
\hline Type I & Walking on straight line with break in the middle. \\
\hline Type II & $\begin{array}{l}\text { Walking on straight line with break in the middle, } \\
\text { and reduced walking speed for the second part. }\end{array}$ \\
\hline Type III & $\begin{array}{l}\text { Walking forward on straight line with break at } \\
\text { reversal point, then turn around and walking back } \\
\text { on straight line. }\end{array}$ \\
\hline Type IV & $\begin{array}{l}\text { Walking on rectangular trajectory with stop to turn } \\
\text { in corners. }\end{array}$ \\
\hline Type V & $\begin{array}{l}\text { Walking on rectangular trajectory without stop to } \\
\text { turn in corners. }\end{array}$ \\
\hline
\end{tabular}

Table 8 Scenarios for the experiment.

\subsection{Experimental Results}

The results for our PNS for each of the five scenarios from Table 8 are presented in turn. For type I to III the initial orientation is specified prior to the experiments, to exclude influences of magnetic disturbances. These influences are then considered for type IV and type V.

\subsubsection{Type I}

In the first experiment, the scenario is a straight path of $51.1 \mathrm{~m}$. In our measurements, the test person walks at an average speed of $1.18 \mathrm{~m} / \mathrm{s}$ and stands still in the middle for around $8 \mathrm{~s}$. Then, the pedestrian continues to walk to the end of the path. Already within this very simple scenario, the ZUPT and non-ZUPT methods are employed for the INS. As an example, ZUPT is used at the beginning and directly after the single stop, and non-ZUPT is used otherwise.

For the smartwatch, the magnitude of the 
acceleration is illustrated in Fig. 18a. First of all, the waiting phase between $24 \mathrm{~s}$ and $32 \mathrm{~s}$ is visible, where the magnitude of the acceleration is equal to the gravity $\left(\approx 9.8 \mathrm{~m} / \mathrm{s}^{2}\right.$ ). As an illustration, the data between $5 \mathrm{~s}$ and 10s is enlarged in Fig. 18b to illustrate successive walking. The swing phase is marked in green and the stance phase in red, respectively. These are separated by conditions $C_{\text {Stance, } 1}$ (Eq. (25)), $C_{\text {Stance, } 2}$ (Eq. (28)) and $C_{\text {Stance,3 }}$ (Eq. (32)). The black rectangles denote wrong detections of stance phases, when only these three conditions are applied.

Fig. 19 illustrates the associated angular velocity. Just as before, the stop of the pedestrian is visible, since the angular velocity stays around $0 \mathrm{rad} / \mathrm{s}$ between 24s and 32s. Again, the detected swing and stance phases are marked, using only the aforementioned conditions $C_{\text {Stance, } 1}, C_{\text {Stance, } 2}$ and $C_{\text {Stance, } 3}$. In addition, wrong detections of stance phases are indicated by black rectangles. Fig. 20 shows the associated angular velocity along the $\mathrm{z}$-axis, where we have included the black rectangles for convenience. It is obvious that the angular velocity along the $\mathrm{z}$-axis is approximately $0 \mathrm{rad} / \mathrm{s}$. Hence, these wrong stance phase detections can be ruled out by $C_{\mathrm{Stance}, 5}$. Similar considerations can be made for $C_{\mathrm{Stance}, 4}$. This justifies the introduction of the additional conditions in Fig. 11 for our PNS. For the conditions $C_{\text {Stance, } 1}$ to $C_{\text {Stance, } 5 \text {, }}$ Fig. 21 illustrates the detection of the stance phase. The individual steps are clearly separable. Moreover, no detection errors occur. Fig. 21b enables to assess the relation between the duration of the swing and stance phase. In respect to the picture, as a rough estimate, the strides should be considered non-successive if the duration of the stance phase is longer than $3 / 2$ times the length of the swing phase, which is in accordance to Eq. (64). The magnitude of the velocity is shown in Fig. 22. Although it is hard to see, the velocity for a new stride is only $0 \mathrm{~m} / \mathrm{s}$ at around $5 \mathrm{~s}$ and 33s. This is because of ZUPT, which is only used when the strides are non-successive. In all other cases a small positive value remains due to non-ZUPT.

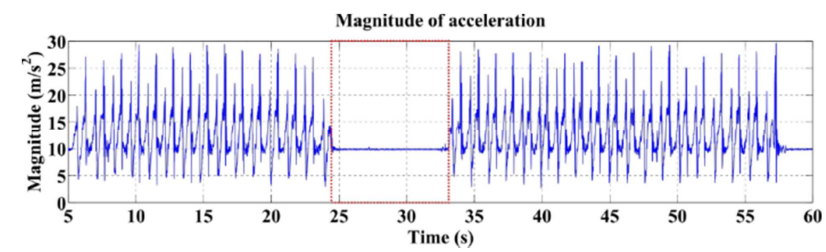

(a) Full time span

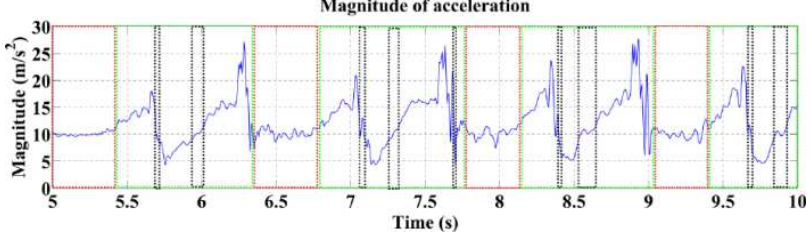

(b) Selected extract

Fig. 18 Measured magnitude of acceleration.

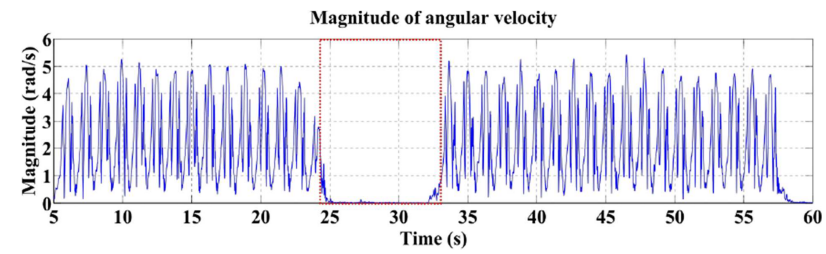

(a) Full time span

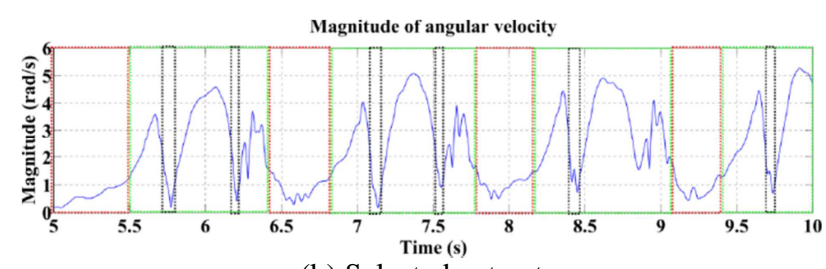

(b) Selected extract

Fig. 19 Measured magnitude of angular velocity.

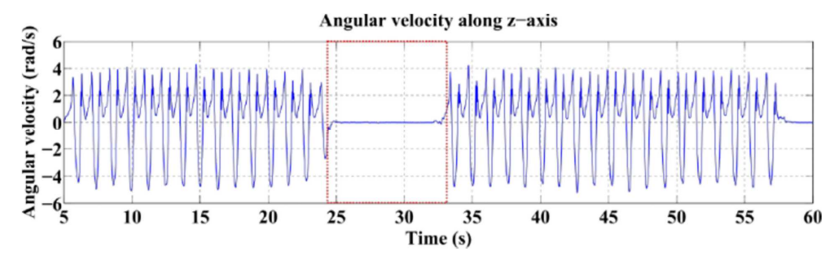

(a) Full time span

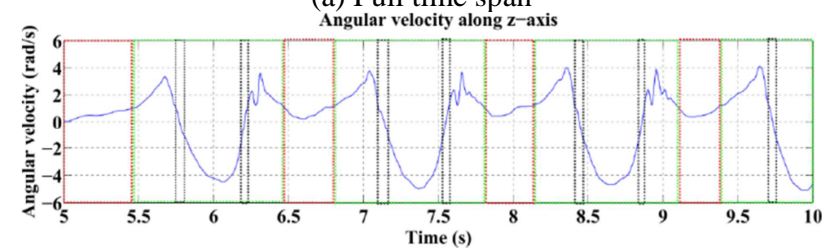

(b) Selected extract

Fig. 20 Measured magnitude of angular velocity along the z-axis. 


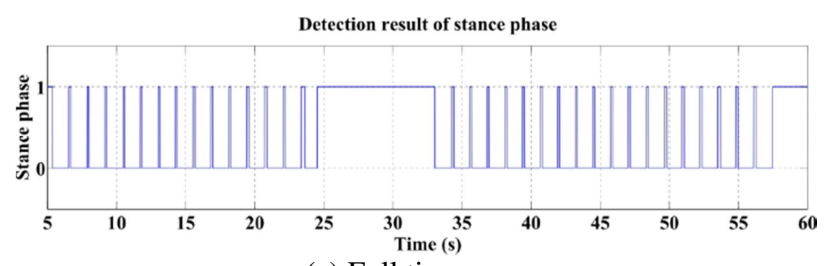

(a) Full time span

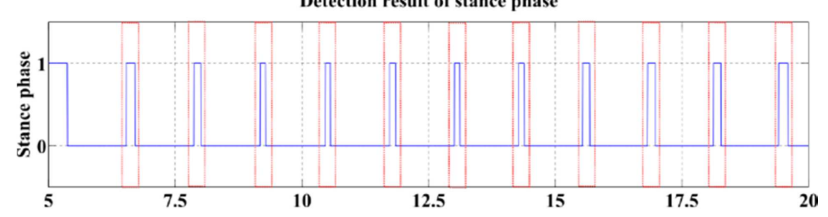

(b) Selected extract

Fig. 21 Derived swing and stance phase.

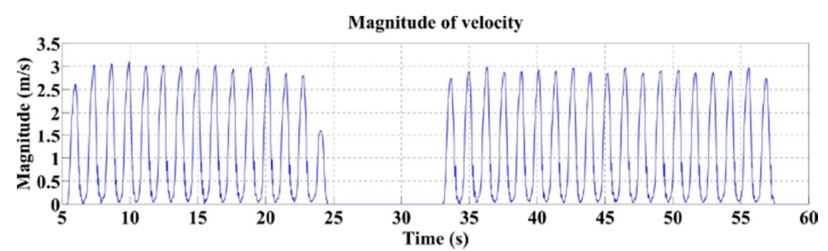

(a) Full time span

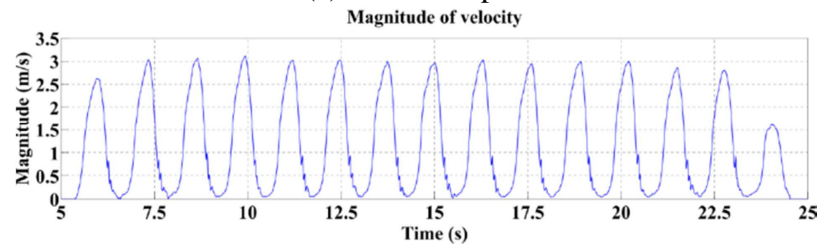

(b) Selected extract

Fig. 22 Measured magnitude of velocity.

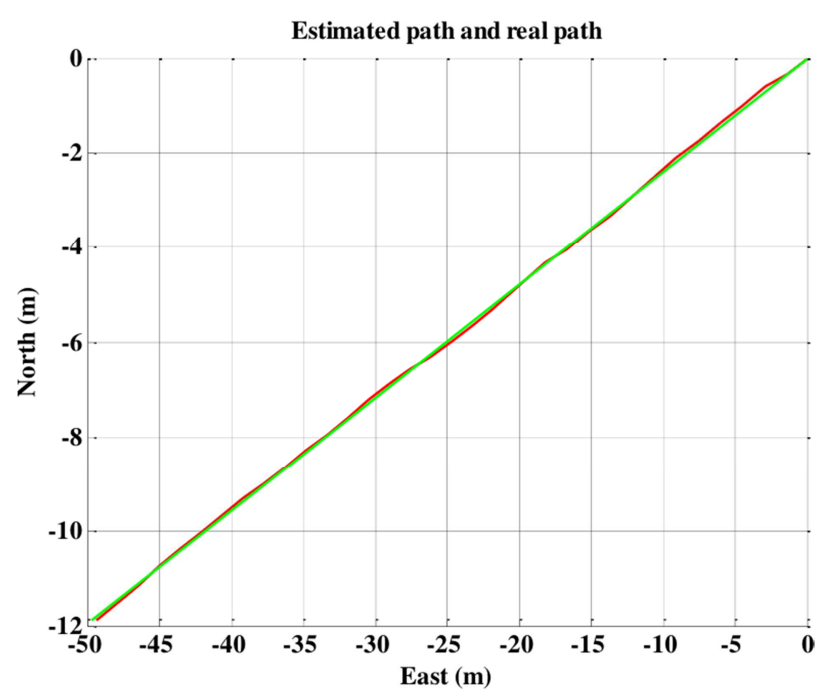

Fig. 23 Estimated path and real path.

The trajectory of the estimated path (red line) in comparison to the real path (green line) is illustrated in Fig. 23. Both trajectories are nearly the same, which is a first indicator for the quality of our PNS. The final position error relative to the TTD is $e_{T T D}=0.5 \%$. In contrast, the results reported in Ref. [32] show an error of $3.6 \%$, which is significantly worse. There, only non-ZUPT is considered, which might be one reason for the differences.

\subsubsection{Type II}

The scenario for type II is exactly the same as for type I, except that the speed is reduced for the last part of the path. To be more precise, the user starts walking at a speed of $1.28 \mathrm{~m} / \mathrm{s}$, stops in the middle for about $5 \mathrm{~s}$ and continues walking at $0.90 \mathrm{~m} / \mathrm{s}$. Fig. 24 shows the detection of the stance phase. It is obvious that for reduced velocity, the duration of the stance phase increases, as it is expected. As before, if we would only use conditions $C_{\text {Stance, } 1}$ to $C_{\text {Stance, } 3}$, the stance phases could not be reliably detected (see Fig. 25).

The trajectory is shown in Fig. 26. The red and blue lines, which are the estimated path for the first and second period, correspond well with the real trajectory (green line). The final position error is $e_{T T D}=0.3 \%$.

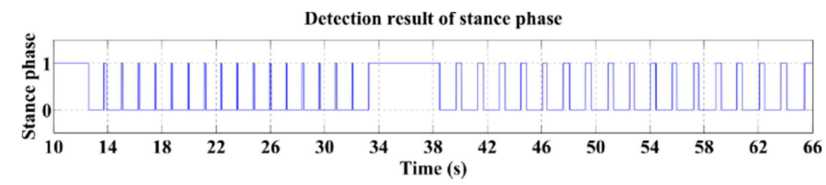

(a) Full time span

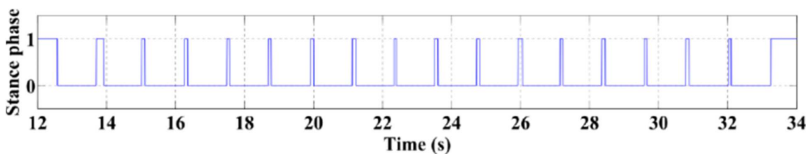

(b) First part

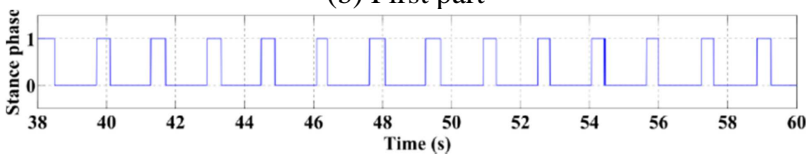

(c) Last part

Fig. 24 Derived swing and stance phase.

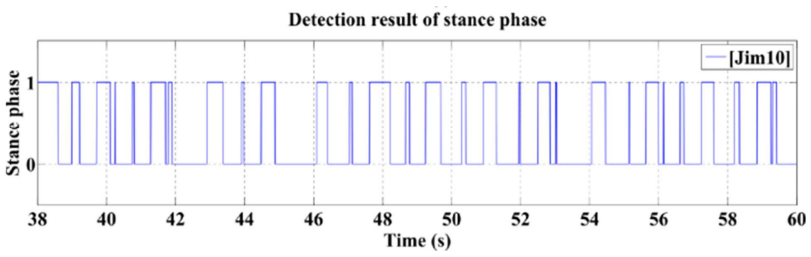

Fig. 25 Non reliable stance phase detection according to Ref. [17]. 


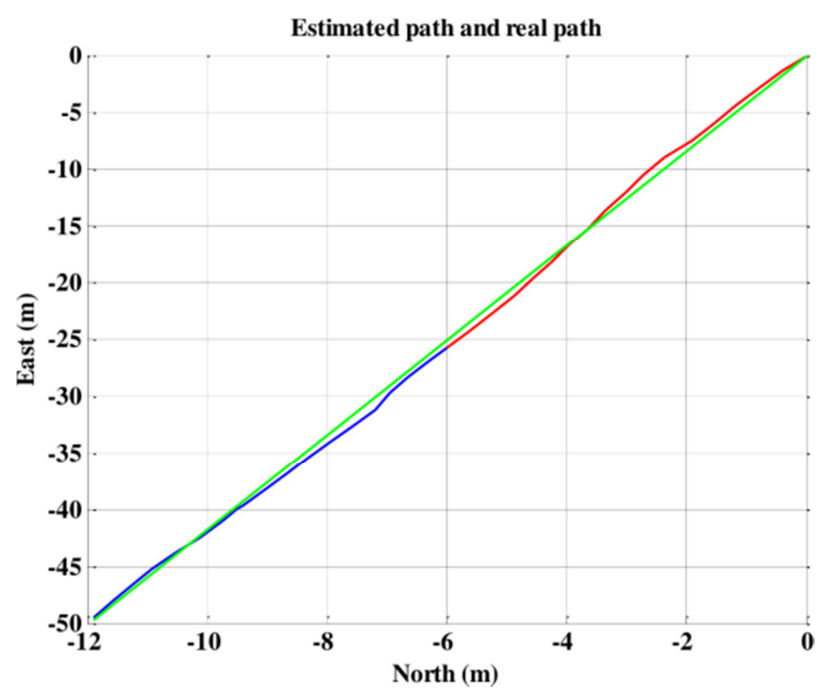

Fig. 26 Estimated path and real path.

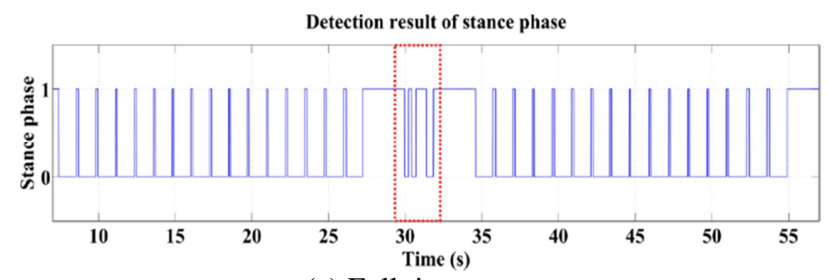

(a) Full time span

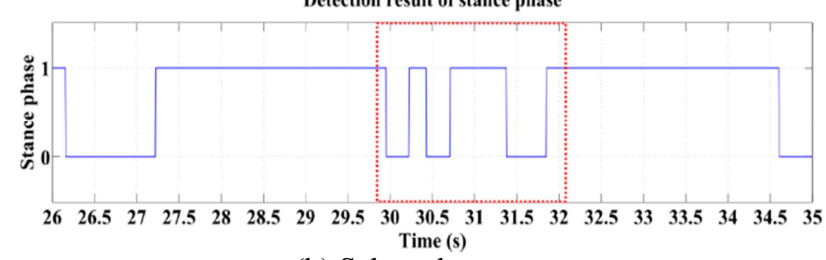

(b) Selected extract

Fig. 27 Derived stance phase detections including wrong detections.

\subsubsection{Type III}

For the third type, the pedestrian walks on a straight path, stands still at the end, turns around and walks back to the origin. For the concrete experiment presented below, the path has a length of $24.4 \mathrm{~m}$ (one way) and the user walks at an average speed of $1.20 \mathrm{~m} / \mathrm{s}$. The detection of the stance phases is depicted in Fig. 27. By using our enhanced conditions, within the time interval [30s, 33s], still three gaits are identified, which are incorrect detections. These detected gaits are caused by moving the ankle during turning. Of course, these gaits also appear for the non-enhanced conditions. For these three wrong gaits, the estimated lengths by only considering the INS are
$5 \mathrm{~mm}, 1.4 \mathrm{~cm}$ and $2.3 \mathrm{~cm}$, respectively. The associated calculated step lengths by only employing the PDR are $1.05 \mathrm{~m}, 0.99 \mathrm{~m}$ and $1.12 \mathrm{~m}$, respectively. This mismatch between the values of the INS and the PDR shows that these are incorrect detections. For this reason, we have included the condition for $\alpha$ in Table 7. For these gaits, it holds that $\frac{\left.\right|^{N} \boldsymbol{r}_{k, I N S} \mid}{\left|{ }^{N} \boldsymbol{r}_{k, P D R}\right|}<0.05 \ll 0.8$. Thus, $\alpha$ is set to one. In doing so, the PDR results are ignored according to Eq. (70).

Fig. 28 shows the trajectory, where the red and blue lines are the estimated paths for the one-way and return trip, respectively. Once more, the green colour denotes the real trajectory. The results indicate that the estimated path corresponds well to the true path. The final position error is about $0.26 \mathrm{~m}$, which implies $e_{T T D}=0.54 \%$. Repeating the experiment five times, as shown in Table 9, produces an average of $e_{T T D}=1.11 \%$.

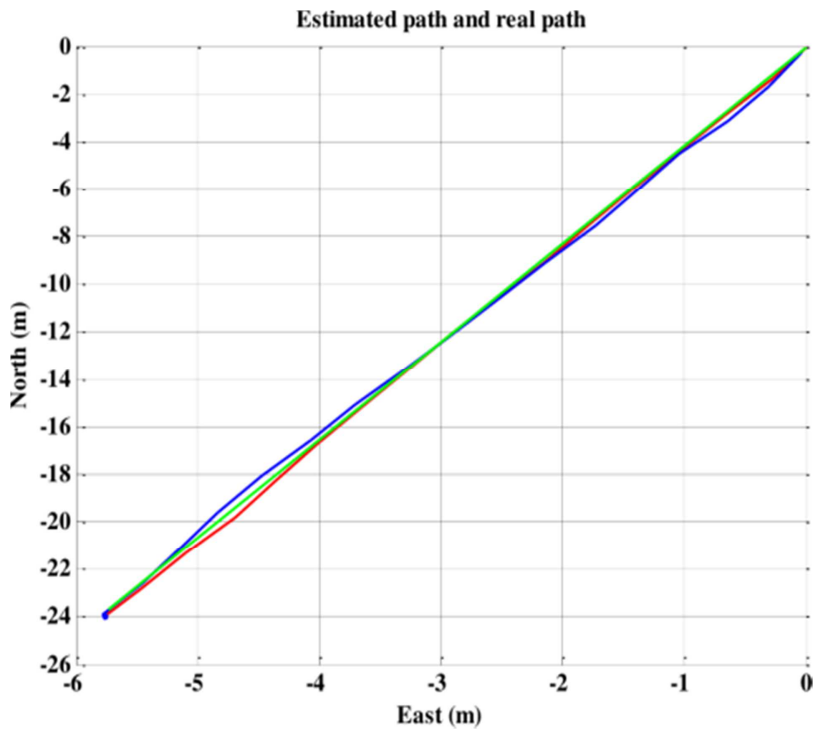

Fig. 28 Estimated path and real path.

Table 9 Data series for type III experiment.

\begin{tabular}{ll}
\hline Experiment & Specification \\
\hline Walk 1 & $0.54 \%$ \\
Walk 2 & $1.62 \%$ \\
Walk 3 & $1.22 \%$ \\
Walk 4 & $0.84 \%$ \\
Walk 5 & $1.35 \%$ \\
Average & $1.11 \%$ \\
\hline
\end{tabular}


This is well below state-of-the-art approaches, e.g. 5\% presented in Ref. [15].

\subsubsection{Type IV}

All further experiments are oriented on the experiment described in Ref. [15]. Here, the trajectory is rectangular shaped with dimensions $13.2 \mathrm{~m} \times 8.3 \mathrm{~m}$. Hence, the TTD is $43 \mathrm{~m}$. For type IV the user stops in each corner, turns around and continuous to walk. The experiment is repeated five times. Fig. 29 shows the trajectory for one sample. Again, the green lines denote the real path and the red line is the estimated trajectory. As can be seen, the initial heading is quite different. After rotating the estimated path, which is shown in blue, both paths correspond well. Using this path, $e_{T T D}=0.58 \%$. The problem of the initial orientation will be described at the end of this section. Table 10 contains the results for all five experiments after calibrating the initial orientation.

\subsubsection{Type V}

The experiments for type $\mathrm{V}$ are similar to type IV, except that the user walks continuously across the corners. Fig. 30 illustrates the trajectories, where the colours are identical to type IV. In Fig. 30, the trajectories correspond well, which shows that in this case the initial orientation is correct. Compared to Ref. [15], where 5\% error is reported, we could achieve $1.73 \%$, as shown in Table 11 for five repetitions of the experiment. In Ref. [35] an even better result of $0.8 \%$ error is reported. For these measurement however, a superior IMU, i.e. the MTiXsens [37], is utilized which offers better resolution and accuracy. Moreover, the IMU is mounted on the foot, which indicates an easier detection of the stance phases and enables to reset the velocity in each gait.

It remains to characterize the error of the initial heading for type IV, in contrast to type V. The problem is caused by the measured magnetic field, as it is shown in Fig. 31. Although the starting point of these two types of experiments is the same, the value of the magnetic field in type IV $(\approx 10 \mu T)$ is opposite to the value in type $\mathrm{V}(\approx-10 \mu T)$. This results in wrong heading information being applied by the Madgwick algorithm. The root cause can be an additional magnetic field being available at the time when the experiment of type IV was executed. One possibility to solve this issue is by incorporating additional information, e.g. by using the orientation vector of another positioning system.

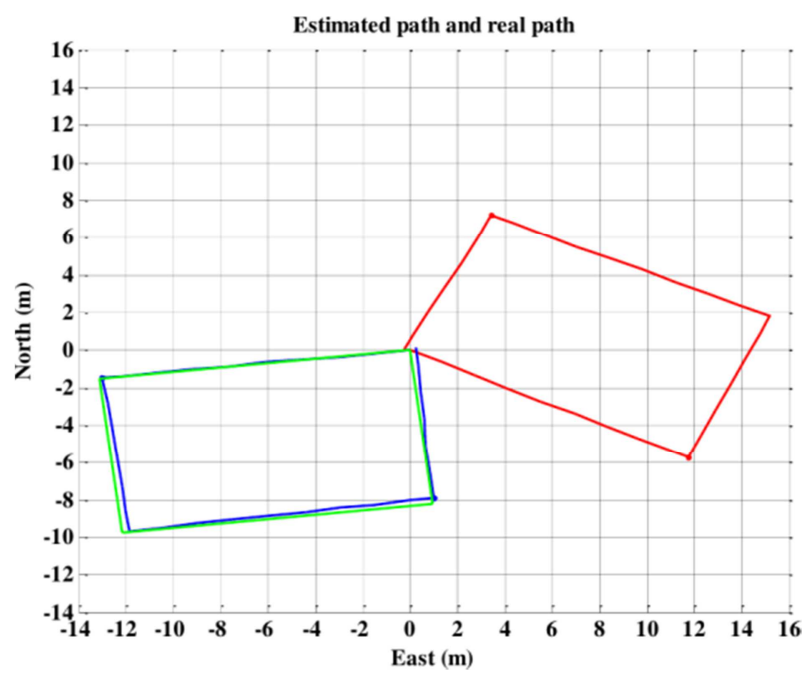

Fig. 29 Estimated path and real path.

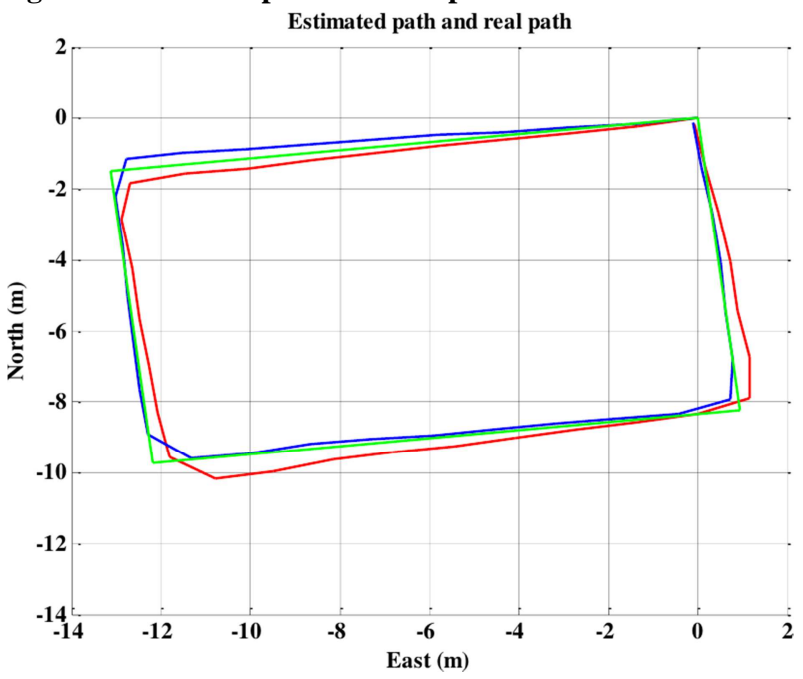

Fig. 30 Estimated path and real path.

Table 10 Data series for type IV experiment with additional calibration of initial orientation.

\begin{tabular}{ll}
\hline Experiment & Specification \\
\hline Walk 1 & $2.82 \%$ \\
Walk 2 & $1.52 \%$ \\
Walk 3 & $0.90 \%$ \\
Walk 4 & $2.68 \%$ \\
Walk 5 & $0.58 \%$ \\
Average & $1.66 \%$ \\
\hline
\end{tabular}


Table 11 Data series for type $V$ experiment with additional calibration of initial orientation.

\begin{tabular}{ll}
\hline Experiment & Specification \\
\hline Walk 1 & $1.72 \%$ \\
Walk 2 & $0.42 \%$ \\
Walk 3 & $2.70 \%$ \\
Walk 4 & $0.35 \%$ \\
Walk 5 & $3.47 \%$ \\
Average & $1.73 \%$ \\
\hline
\end{tabular}

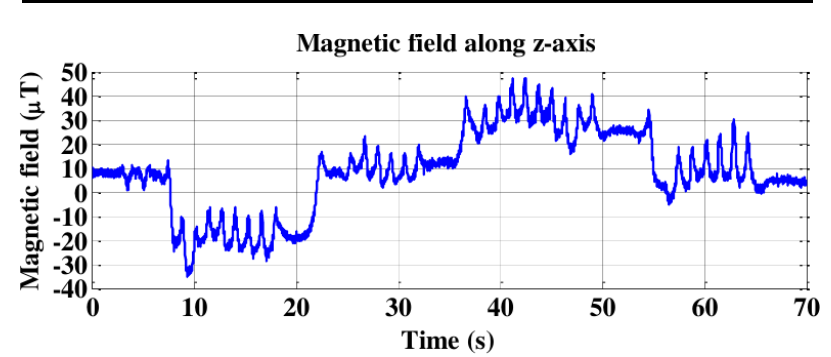

(a) Example from Type IV Magnetic field along z-axis

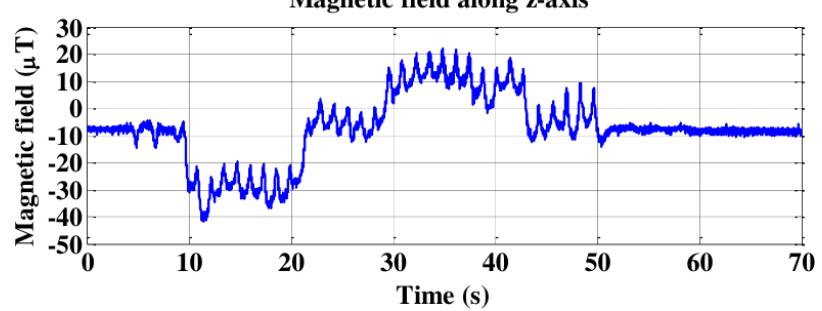

(b) Example from Type $\mathrm{V}$

Fig. 31 Measured magnetic field along the z-axis.

\section{Conclusion}

The experiments have shown that our PNS fulfils the requirements on an indoor positioning system. First of all, it is cheap and does not require any additional hardware, except a smartphone and a smartwatch. Our results keep pace with state-of-the-art, whereby we outperform most approaches, except Ref. [35]. The latter however utilizes a better IMU, with superior sensors. For our measurements, we have shown that the error for the straight line experiments is below $1 \%$. For all other scenarios, the average error is always below $2 \%$, if we do not consider the initial orientation error due to magnetic disturbances. Nevertheless, our system cannot fully resolve the drawback of all INS/PDR, i.e. the accumulation of errors over time. Having said this, our system offers a very good performance since it overcomes two main error sources of common INS: wrong turning angles and too long/too short estimated path lengths. Hence, it is at very well suited as a bridging technology for seamless positioning.

\section{Acknowledgement}

The research leading to these results has received funding from the European Community's Seventh Framework Programme (FP7/2007-2013) under grant agreement ICT-FP7-611526 (MAGELLAN).

\section{References}

[1] Gunia, M., Protze, F., Joram, N., and Ellinger, F. 2016. "Setting Up an Ultra-wideband Positioning System Using Off-the-Shelf Components. "13th Workshop on Positioning, Navigation and Communication (WPNC), 1-6.

[2] Joram, N., Al-Qudsi, B., Wagner, J., Strobel, A., and Ellinger, F. 2013. "Design of a Multi-band FMCW Radar Module. "10th Workshop on Positioning Navigation and Communication (WPNC), 1-6.

[3] Mazilu, S., and Tröster, G. 2015. "A Study on Using Ambient Sensors from Smartphones for Indoor Location Detection. "12th Workshop on Positioning Navigation and Communication (WPNC), 1-6.

[4] Titterton, D., and Weston, J. 2004. Strapdown Inertial Navigation Technology. The American Institute of Aeronautics and Astronautics, 2nd edition.

[5] Fischer, W.J. 2000. "Mikrosystemtechnik." Vogel Fachbuch.

[6] Woodman. 2007. "An Introduction to Inertial Navigation. "Technical Report, University of Cambridge.

[7] Madgwick, S. O. H., Harrison, A. J. L., and Vaidyanathan, R. 2011. "Estimation of IMU and MARG Orientation Using a Gradient Descent Algorithm. "IEEE International Conference on Rehabilitation Robotics, Zurich.

[8] Lunze, K. 1991. "Einführung in Die Elektrotechnik." Verlag Technik GmbH Berlin.

[9] Groves, P. D. 2013. "Principles of GNSS, Inertial and Multisensor Integrated Navigation Systems." Artech House, 2nd edition.

[10] Cuccurullo, S. J. 2014. "Physical Medicine and Rehabilitations Board Review." Demos Medical Publishing, 409-11.

[11] Kuipers, J. B. 2002. "Quaternions and Rotation Sequences: A Primer with Applications to Orbits, Aerospace and Virtual Reality." Princeton University Press.

[12] Eberly, D. 2016. "Quaternion Algebra and Calculus." Create Notes. 
[13] Jia, Y.-B. 2016. "Quaternions and Rotation.” Com. S 477/577 Notes.

[14] Ioffe, M. "Quaternion Differentiation." https://www.euclideanspace.com/physics/kinematics/ang ularvelocity/QuaternionDifferentiation2.pdf.

[15] Edwan, E., Gunia, M., Al-Qudsi, B., Joram, N., and Ellinger, F. 2015. "Smart Wearable Combined with Smart Phones for Pedestrian Navigation Applications." 12th Workshop on Positioning Navigation and Communication (WPNC), 1-6.

[16] Callmer, J. 2013. "Autonomous Localization in Unkown Environments." Dissertation, Sweden.

[17] Jiménez, A. R., Seco, F., Prieto, J. C. ,and Guevara, J. 2010. "Indoor Pedestrian Navigation Using an INS/EKF Framework for Yaw Drift Reduction and a Foot-Mounted IMU." 7th Workshop on Positioning Navigation and Communication (WPNC), 135-43.

[18] Mood, A. M., Graybill, F., and Boes, D. C. 1974. "Introduction to the Theory of Statistics." $M c G R A W-H I L L, 3$ rd edition.

[19] Weinberg, H. 2002. "Using the ADXL202 in Pedometer and Personal Navigation Applications." Analog Devices AN-602 Application Note2 (2): 1-6.

[20] Wiebren, Z., and Hof, A. L. 1997. "Displacement of the Pelvis during Human Walking: Experimental Data and Model Predictions." Gait \& Posture 6 (3): 249-62.

[21] Alvarez, D., Gonzalez, R. C., and Lopez, A. 2006. "Comparison of Step Length Estimators from Wearable Accelerometer Devices." 2006 International Conference of the IEEE Engineering in Medicine and Biology Society, 5964-7.

[22] Jahn, J., Batzer, U., Seitz, J., Patino-Studencka, L., and Gutiérrez Boronat, J. 2010. "Comparison and Evaluation of Acceleration Based Step Length Estimators for Handheld Devices." Indoor Positioning and Indoor Navigation, 1-6.

[23] Renaudin, V., Susi, M., and Lachapelle, G. 2012. "Step Length Estimation Using Handheld Inertial Sensors." Sensors 12 (7): 8507-25.

[24] Wahba, G. "A Least-Square Estimate of Spacecraft Attitude." SIAM Rev. 7, 409.

[25] Markley, F. L., and Mortari, D. 1999. "How to Estimate
Attitude from Vector Observations." AAS/AIAA Astrodynamics Specialist Conf. AAS, 99-427.

[26] Valenti, G. R., Dryanovski, I., and Xiao, J. 2015. "Keeping a Good Attitude: A Quaternion-based Orientation Filter for IMUs and MARGs." Sensors 2015, 15 (8):19302-30.

[27] Shuster, M. D., and Oh, S. D. 1981. "Three-Axis Attitude Determination from Vector Observations." Journal of Guidance and Control.

[28] Sabatini, A. M. 2006. "Quaternion-Based Extended Kalman Filter for Determining Orientation by Inertial and Magnetic Sensing." IEEE Transactions on Biomedical Engineering 53.7, pp.1346-56.

[29] Xsens Technologies B.V.2009. "MTi and MTx User Manual and Technical Documentation."

[30] Micro-strain Inc. 2009. "3DM-GX $3^{\circledR}-25$ Miniature Attitude Heading Reference Sensors."

[31] National Centers for Environmental Information. 2017. "Magnetic Field Calculators." https://www.ngdc.noaa.gov/geomag-web/\#igrfwmm.

[32] Wu, X., Wang, Y., and Pottie, G. 2014. "A Non-zupt Gait Reconstruction Method for Ankle Sensors." 36th Annual Int. Conf. on the IEEE Eng. in Medicine and Biology Society, 5884-7.

[33] Patterson, M. R., Delahunt, E., Sweeney, K. T., and Caulfield, B. 2014. "An Ambulatory Method of Identifying Anterior Cruciate Ligament Reconstructed Gait Patterns." Sensors (Basel), 887-99.

[34] Michel, T., Fourati, H., Genevès, P., and Layaïda, N. 2015. "A Comparative Analysis of Attitude Estimation for Pedestrian Navigation with Smartphones." IEEE Int. Conf. on Indoor Positioning and Indoor Navigation.

[35] Fourati, H. 2015. "Heterogeneous Data Fusion Algorithm for Pedestrian Navigation via Foot-Mounted Inertial Measurement Unit and Complementary Filter." IEEE Transactions on Instrumentation and Measurement, 221-9.

[36] Goyal, P. 2011. "Strap-Down Pedestrian Dead-Reckoning System." IEEE Int. Conf. on Indoor Positioning and Indoor Navigation.

[37] Vydhyanathan, A., Bellusci, G., Luinge, H., and Slycke, P. 2015. "The Next Generation Xsens Motion Trackers for Industrial Applications." Version 2.0.1, Xsens. 